\title{
Israel-Stewart Approach to Viscous Dissipative Extended Holographic Ricci Dark Energy Dominated Universe
}

\author{
Surajit Chattopadhyay \\ Pailan College of Management and Technology (MCA Division), Bengal Pailan Park, Kolkata 700 104, India \\ Correspondence should be addressed to Surajit Chattopadhyay; surajitchatto@outlook.com
}

Received 16 April 2016; Revised 24 May 2016; Accepted 27 June 2016

Academic Editor: Elias C. Vagenas

Copyright ( 2016 Surajit Chattopadhyay. This is an open access article distributed under the Creative Commons Attribution License, which permits unrestricted use, distribution, and reproduction in any medium, provided the original work is properly cited. The publication of this article was funded by $\mathrm{SCOAP}^{3}$.

\begin{abstract}
This paper reports a study on the truncated Israel-Stewart formalism for bulk viscosity using the extended holographic Ricci dark energy (EHRDE). Under the consideration that the universe is dominated by EHRDE, the evolution equation for the bulk viscous pressure $\Pi$ in the framework of the truncated Israel-Stewart theory has been taken as $\tau \dot{\Pi}+\Pi=-3 \xi H$, where $\tau$ is the relaxation time and $\xi$ is the bulk viscosity coefficient. Considering effective pressure as a sum of thermodynamic pressure of EHRDE and bulk viscous pressure, it has been observed that under the influence of bulk viscosity the EoS parameter $w_{\mathrm{DE}}$ is behaving like phantom, that is, $w_{\mathrm{DE}} \leq-1$. It has been observed that the magnitude of the effective pressure $p_{\text {eff }}=p+\Pi$ is decaying with time. We also investigated the case for a specific choice of scale factor; namely, $a(t)=\left(t-t_{0}\right)^{\beta /(1-\alpha)}$. For this choice we have observed that a transition from quintessence to phantom is possible for the equation of state parameter. However, the $\Lambda \mathrm{CDM}$ phase is not attainable by the state-finder trajectories for this choice. Finally it has been observed that in both of the cases the generalized second law of thermodynamics is valid for the viscous EHRDE dominated universe enveloped by the apparent horizon.
\end{abstract}

\section{Introduction}

Accelerated expansion of the current universe was reported by Riess et al. [1] of high-redshift supernovae search team and Perlmutter et al. [2] of supernovae cosmology project team through accumulation of observational data from distant type Ia Supernovae. Discovery of $[1,2]$ was truly ground breaking and subsequently this has been further confirmed by other observational studies including more detailed studies of supernovae and independent evidence from clusters of galaxies, large-scale structure (LSS), and the cosmic microwave background (CMB) [3]. The reason behind this expansion is referred to as "dark energy" (DE), which is regarded as an exotic matter characterized by negative pressure and having equation of state parameter $w=p / \rho<-1 / 3$ required for accelerated expansion of the universe. Nature of this $\mathrm{DE}$ is not yet clear and different $\mathrm{DE}$ candidates have been proposed till date. Some remarkable reviews on DE include [4-7]. While proposing a phantom cosmology based unifying approach to early and late-time universe, Nojiri and Odintsov [8] suggested generalized holographic dark energy (HDE) involving infrared cutoff combined with FRW parameters and also discussed the entropy bound in phantom era. HDE is based on "holographic principle" and the density of HDE is [9] $\rho_{\Lambda}=3 c^{2} M_{p}^{2} L^{-2}$, where $L$ is the infrared cutoff. Some notable works on HDE include [10-15]. Furthermore, there exist plethora of literatures on HDE in theoretical aspects as well as observational constraints, for example, [16-18].

In the present work, we consider a special form of HDE [19] dubbed as "extended holographic Ricci dark energy" (EHRDE) [20, 21], whose density has the folowing form:

$$
\rho_{\mathrm{DE}}=3 M_{p}^{2}\left(\alpha H^{2}+\beta \dot{H}\right),
$$

where the upper dot represents derivative with respect to cosmic time $t, M_{p}^{2}$ is the reduced Planck mass, and $\alpha$ and $\beta$ are constants to be determined. In this context we should mention that the role of a distance proportional to the Ricci scale as a causal connection scale for perturbations was noticed in $[22,23]$ and used for the first time as a DE cutoff scale. Detailed cosmology of Ricci DE was discussed in [24] and some further literatures on Ricci DE include [25-28]. 
Wang and $\mathrm{Xu}$ [29] found the best-fit values in order to make the cutoff of [20] to be consistent with observational data as $\alpha=0.8502_{-0.0875-0.1064}^{+0.0984+0.1299}$ and $\beta=0.4817_{-0.0773-0.0955}^{+0.0842+0.176}$. In the current work, we will take $\alpha=0.98$ and $\beta=0.37$. In the early evolution of the universe, dissipative effects, including both bulk and shear viscosity, are supposed to play a very important role [30]. Chimento et al. [31] had shown that accelerated expansion can be derived by the combination of a cosmic fluid with bulk dissipative pressure and quintessence matter and it can also solve the coincidence problem. Evolution of the universe involves a sequence of important dissipative processes that includes GUT phase transition at $t \approx 10^{-34} \mathrm{~s}$ and a temperature of about $T \approx$ $10^{27} \mathrm{~K}$ [30]. Eckart [32] and Landau and Lifshitz [33] were the first ones to attempt to create a theory of relativistic dissipative fluids. A relativistic second-order theory was developed by Israel and Stewart [34].

A time dependent viscosity consideration was made to DE by Nojiri and Odintsov [35] while considering EoS with inhomogeneous, Hubble parameter dependent term and it was demonstrated that the thermodynamic entropy may be positive even in phantom era as a result of crossing the phantom boundary. Pun et al. [30] considered a generalization of the Chaplygin gas model and by assuming the presence of a bulk viscous type dissipative term in the effective thermodynamic pressure of the gas and by considering dissipative effects described by truncated Israel-Stewart model they [30] had shown that viscous Chaplygin gas model offers an effective dynamical possibility for replacing the cosmological constant. Effects of viscosity in Chaplygin gas with different modifications have been studied in [36-38]. Cataldo et al. [39] investigated dissipative processes in the universe in the full causal Israel-Stewart-Hiscock theory and showed that the negative pressure generated by the bulk viscosity cannot avoid the idea that the dark energy of the universe is to be phantom energy. Brevik and Gorbunova [40] assumed the bulk viscosity to be proportional to the scalar expansion in a spatially flat FRW universe and had shown that it could lead the universe to phantom phase even if the universe lies in the quintessence phase in the nonviscous scenario. Ren and Meng [41] considered a generally parameterized EoS in the cosmological evolution with bulk viscosity media modelled as dark fluid. Setare and Sheykhi [42] studied the validity of generalized second law of thermodynamics in the presence of viscous dark energy in a nonflat universe and concluded with validity of the generalized second law. Feng and $\mathrm{Li}$ [43] investigated viscous Ricci dark energy and showed that once viscosity is taken into account, the problem on age of the universe gets alleviated. Amirhashchi and Pradhan [44] considered viscous and nonviscous dark energy EoS parameter in anisotropic Bianchi type I space-time and could get a transition from quintessence to phantom. Brevik et al. [45] investigated interacting dark energy and dark matter in flat FRW universe taking bulk viscosity as a function of Hubble parameter $H$ and cosmic time $t$ and studied subsequent corrections of thermodynamic parameters. Velten et al. [46] derived conditions under which either viscous matter or radiation cosmologies can be mapped into the phantom dark energy scenario with constraints from multiple observational data sets. Jamil and Farooq [47] presented a generalization of interacting HDE using the viscous generalized Chaplygin gas and reconstructed the potential and the dynamics of the scalar field. Setare and Kamali [48] study warm-viscous inflationary universe model on the brane in a tachyon field theory and obtained the general conditions which are required for this model to be realizable. In a very recent work, Bamba and Odintsov [49] investigated a fluid model in which EoS for a fluid includes bulk viscosity and found that the spectral index of the curvature perturbations, the tensor-to-scalar ratio of the density perturbations, and the running of the spectral index can be consistent with the recent Planck results. It may be noted that in a remarkable work Brevik et al. [50] discussed entropy of DE filled FRW universe in the framework of holographic Cardy-Verlinde formula and expressed entropy in terms of energy and Casimir energy depending on the EoS and, in a relatively recent work, Brevik et al. [51] derived a formula for the entropy for a multicomponent coupled fluid that under certain conditions may reduce to the CardyVerlinde form to relate the entropy of a closed FRW universe to the energy contained in it together with its Casimir energy. In some recent studies, bulk viscosity has been incorporated in the studies of modified gravity too and studies in this direction include [52-54].

Plan of the present work is as follows. In Section 2 we will apply Israel-Stewart theory to study the behavior of viscous extended holographic Ricci dark energy (EHRDE) without any specific choice of scale factor as well as the choice $a(t)=\left(t-t_{0}\right)^{\beta /(1-\alpha)}$. In Section 3 we will study the statefinder parameters and investigate whether $\Lambda$ CDM phase is attainable for both the cases. In Section 4 we will examine validity of the generalized second law of thermodynamics for both the cases and in Section 5 we will conclude the paper.

\section{Israel-Stewart Approach}

2.1. Israel-Stewart Approach without Any Specific Choice of Scale Factor. In the cosmological framework bulk viscosity can be thought of as an internal friction due to the different cooling rates in an expanding gas [30]. As the dissipation due to bulk viscosity converts kinetic energy of the particles into heat, the effective pressure is expected to be reduced in an expanding fluid. For flat homogeneous FriedmannRobertson-Walker (FRW) with a line element

$$
d s^{2}=d t^{2}-a^{2}(t)\left(d x^{2}+d y^{2}+d z^{2}\right)
$$

filled with a bulk viscous cosmological fluid, the energymomentum tensor is given by [30]

$$
T_{i}^{k}=(\rho+p+\Pi) u_{i} u^{k}-(p+\Pi) \delta_{i}^{k},
$$

where $\rho, p$, and $\Pi$ are energy density, thermodynamic pressure, and the bulk viscous pressure, respectively. $u_{i}$ is for velocity that satisfies the condition $u_{i} u^{i}=1$. Here, $N^{i}=$ $n u^{i}$ and $S^{i}=\sigma N^{i}-\left(\tau \Pi^{2} / 2 \xi T\right) u^{i}$ are particle and entropy fluxes, respectively, where $n, \sigma$, and $T \geq 0$ imply the number density, the specific entropy, and temperature, respectively. Also, bulk viscosity coefficient and relaxation time are $\xi$ and 
$\tau \geq 0$, respectively. If the Hubble parameter is $H=\dot{a} / a$, then the gravitational field equations together with the continuity equation are [30]

$$
\begin{gathered}
3 H^{2}=\rho, \\
2 \dot{H}+3 H^{2}=-p-\Pi, \\
\dot{\rho}+3 H(\rho+p)=-3 H \Pi .
\end{gathered}
$$

The effect of the bulk viscosity can be considered by adding thermodynamic pressure $p$ to the bulk viscous pressure $\Pi$; that is,

$$
p_{\text {eff }}=p+\Pi
$$

terms in the energy-momentum tensor. Taking $x=\ln a$ in (1), we have $\left(M_{p}^{2}=1\right)$;

$$
\rho_{\mathrm{DE}}=3\left(\alpha H^{2}+\frac{\beta}{2} \frac{d H^{2}}{d x}\right) .
$$

Considering $\rho=\rho_{\mathrm{DE}}$ in (4), we have the Hubble parameter

$$
H(x)=H_{0} \exp \left(\frac{x(1-\alpha)}{\beta}\right) .
$$

Putting $x=\ln a$ in (9), we can write

$$
H=H_{0} a^{(1-\alpha) / \beta},
$$

which can be written in the form of a differential equation as follows:

$$
\dot{a}(t)=H_{0} a(t)^{1+(1-\alpha) / \beta},
$$

where the upper dot implies time derivative with respect to cosmic time $t$. Since for the current time $t=t_{0}$ we have $a=1$, we have the following particular solution for $a(t)$ :

$$
a(t)=\left(\frac{H_{0}\left(t-t_{0}\right)(-1+\alpha)+\beta}{\beta}\right)^{\beta /(-1+\alpha)} .
$$

Hence, Hubble parameter as expressed in (9) can be written as a function of $t$ :

$$
H=\frac{H_{0} \beta}{H_{0}\left(t-t_{0}\right)(-1+\alpha)+\beta} .
$$

Using (13) in (1), we have the reconstructed RDE as

$$
\rho_{\mathrm{DE}}=3 H^{2}=\frac{3 H_{0}^{2} \beta^{2}}{\left(H_{0}\left(t-t_{0}\right)(-1+\alpha)+\beta\right)^{2}} .
$$

Using (14) in (6), we have the EoS parameter

$$
\begin{aligned}
& w_{\mathrm{DE}}=-1 \\
& +\frac{1}{3}\left(-\frac{2}{\beta}+\frac{2 \alpha}{\beta}-\frac{\Pi\left(H_{0}\left(t-t_{0}\right)(-1+\alpha)+\beta\right)^{2}}{H_{0}^{2} \beta^{2}}\right) .
\end{aligned}
$$

Hence, for present acceleration, that is, $w<-1 / 3$, at $t=t_{0}$ we need

$$
\alpha<1+\frac{\beta}{2}\left(6+\frac{\Pi\left(t=t_{0}\right)}{H_{0}^{2}}\right) .
$$

If the current universe is in phantom phase, that is, $w<-1$, then we will require

$$
\alpha<1+\frac{\Pi\left(t=t_{0}\right)}{2 H_{0}^{2}} \beta .
$$

For the bulk viscosity coefficient $\xi$ and for the relaxation time $\tau$ of the viscous extended holographic Ricci dark energy, we assume the following phenomenological laws [30]:

$$
\begin{aligned}
& \xi=\eta \rho^{\nu}, \\
& \tau=\xi \rho^{-1}=\eta \rho^{\nu-1},
\end{aligned}
$$

where $\eta \geq 0$ and $v \geq 0$ are constants. At this juncture, it may be stated that the bulk viscosity coefficient $\xi$ is being considered as a function of $\rho(t)$. Hence, possibility is open to a variety of $\xi(\rho(t))$. The case $\nu=1 / 2$ yields $\xi=\eta \rho^{1 / 2}$ that corresponds to a power-law expansion for the scale factor. To obtain solution with Big Rip, no restriction is imposed on $\nu$ and similar approach was adopted in [39]. Another work that is noteworthy in this context is done by Colistete Jr. et al. [55], where for viscous generalized Chaplygin gas a variety of solutions were presented for different ranges of $\nu$. Taking $\nu=2$ and subsequently solving the evolution equation for $\Pi$ in the framework of the truncated Israel-Stewart theory given by

$$
\tau \dot{\Pi}+\Pi=-3 H \xi,
$$

we obtain the evolution equation of the bulk viscous pressure as

$$
\begin{aligned}
\Pi & =e^{-\left(\varphi_{1} t+\varphi_{2}\right)^{3} / 9 \varphi_{2} H_{0}^{2} \eta} C_{1} \\
& -e^{-\left(\varphi_{1} t+\varphi_{2}\right)^{3} / 9 \varphi_{2} H_{0}^{2} \eta} H_{0}^{3}\left(-9 e^{\left(\varphi_{1} t+\varphi_{2}\right)^{3} / 9 \varphi_{2} H_{0}^{2} \eta}\right. \\
& \left.+3^{2 / 3}\left(-\frac{\left(\varphi_{1} t+\varphi_{2}\right)^{3}}{\varphi_{2} H_{0}^{2} \eta}\right)^{2 / 3} \Gamma\left[\frac{1}{3},-\frac{\left(\varphi_{1} t+\varphi_{2}\right)^{3}}{9 \varphi_{2} H_{0}^{2} \eta}\right]\right) \\
& \cdot\left(2 \varphi_{2}\left(\varphi_{1} t+\varphi_{2}\right)^{2}\right)^{-1},
\end{aligned}
$$

where

$$
\begin{aligned}
\varphi_{1} & =\frac{H_{0}}{\beta}(\alpha-1), \\
\varphi_{2} & =1+\frac{H_{0} t_{0}}{\beta}(1-\alpha) .
\end{aligned}
$$


And $C_{1}$ is the constant of integration. Hence, EoS parameter, when expressed as a function of $x(=\ln a)$, takes the form

$$
\begin{gathered}
w_{\mathrm{DE}}=-1+\frac{1}{3}\left(\frac{2(\alpha-1)}{\beta}-\frac{1}{2 H_{0}^{2}} e^{2 x(\alpha-1) / \beta-X}(2\right. \\
-H_{0}^{3} \beta\left(-9 C_{1} e^{X}+3^{2 / 3}(-X)^{2 / 3} \Gamma\left[\frac{1}{3},-X\right]\right) \\
\cdot\left(H_{0}\left(t_{0}(1-\alpha)+\beta\right)\right) \\
\left.\left.\left.\cdot\left(\frac{X\left(9 H_{0}^{2}\left(H_{0} t_{0}(1-\alpha)+\beta\right) \eta\right)}{\beta}\right)^{2 / 3}\right)^{-1}\right)\right)
\end{gathered}
$$

where

$$
\begin{gathered}
X=\beta\left(\frac{H_{0}(\alpha-1)}{\beta}+\left(1-\frac{H_{0} t_{0}(\alpha-1)}{\beta}\right)\right. \\
\left.\cdot\left(t_{0}+\frac{\left(-1+e^{x(\alpha-1) / \beta}\right) \beta}{H_{0}(\alpha-1)}\right)\right)^{3}
\end{gathered}
$$

$$
\cdot\left(H_{0}^{2}\left(H_{0} t_{0}(1-\alpha)+\beta\right) \eta\right)^{-1}
$$

Equation (22) imposes one more constraint on the relationship between $\alpha$ and $\beta$ as

$$
\alpha \neq 1+\frac{\beta}{H_{0} t_{0}}
$$

We would like to add a note at this juncture. Equation (18) comes as a phenomenological law for bulk viscosity coefficient and relaxation time with $\eta \geq 0$; that is, possibility of 0 is not excluded. Clearly, for $\eta=0$, the bulk viscous pressure will vanish and we will get back the nonviscous scenario. However, it is clear that the evolution equation for $\Pi$ in the framework of Israel-Stewart theory is written for viscous scenario and for nonviscous scenario it is irrelevant. Equation (19) is a linear differential equation on $\Pi$ whose integrating factor is exp $\int(d t / \tau)$ and obviously the integrating factor will not exist if $\eta=0$ and hence there will exist no solution and (23) will be of no existence.

From (22) we observe that for $\alpha=0.98$ and $\beta=0.37$ in EHRDE as mentioned in Section 1 we have for very late stage of the universe

$$
\begin{aligned}
& x \longrightarrow \infty \Longrightarrow \\
& w_{\mathrm{DE}} \longrightarrow-1+\frac{1}{3}\left(-0.108-\frac{1}{H_{0}^{2}}-\left(1 0 ^ { - 5 } H _ { 0 } ^ { 3 } \left(-9+19577.259\left(\frac{\left(0.137+0.0148 H_{0} t_{0}+4 \times 10^{-4} H_{0}^{2}\left(-1+t_{0}^{2}\right)\right)^{3}}{H_{0}^{5}\left(-0.37-0.02 H_{0} t_{0}\right) \eta}\right)\right.\right.\right. \\
& \left.\cdot \Gamma\left[\frac{1}{3}, \frac{101452.804\left(0.1369+0.0148 H_{0} t_{0}+4 \times 10^{-4} H_{0}^{2}\left(t_{0}^{2}-1\right)\right)^{3}}{H_{0}^{5}\left(-0.37-0.02 H_{0} t_{0}\right) \eta}\right)\right)\left(\left(-0.37-0.02 H_{0} t_{0}\right)(0.1369\right. \\
& \left.\left.\left.+0.0148 H_{0} t_{0}+4 \times 10^{-4} H_{0}^{2}\left(t_{0}^{2}-1\right)\right)^{2}\right)^{-1}\right)
\end{aligned}
$$

and for early stage

$$
\begin{aligned}
& x \longrightarrow-0.14 \Longrightarrow \\
& w_{\mathrm{DE}} \longrightarrow-1+\frac{1}{3} \\
& \\
& \cdot\left(-\frac{1}{H_{0}^{2}} 0.5 e^{-0.04\left(-0.14+0.99 H_{0} t_{0}+H_{0}^{2}\left(-0.054+0.054 t_{0}^{2}\right)\right)^{3} / H_{0}^{5}\left(0.37+0.02 H_{0} t_{0}\right) \eta}\right. \\
& \cdot\left(2-\left(0 . 3 7 H _ { 0 } ^ { 3 } \left(-9 e^{0.04\left(-0.14+0.99 H_{0} t_{0}+H_{0}^{2}\left(-0.054+0.054 t_{0}^{2}\right)\right)^{3} / H_{0}^{5}\left(0.37+0.02 H_{0} t_{0}\right) \eta}\right.\right.\right.
\end{aligned}
$$




$$
\begin{aligned}
& +1.07\left(-\frac{\left(-0.14+0.99 H_{0} t_{0}+H_{0}^{2}\left(-0.054+0.054 t_{0}^{2}\right)\right)^{3}}{H_{0}^{5}\left(0.37+0.02 H_{0} t_{0}\right) \eta}\right)^{2 / 3} \\
& \left.\left.\cdot \Gamma\left[0.33,-\frac{0.04\left(-0.054 H_{0}+\left(-0.14 / H_{0}+t_{0}\right)\left(1+0.054 H_{0} t_{0}\right)\right)^{3}}{H_{0}^{2}\left(0.37+0.02 H_{0} t_{0}\right) \eta}\right]\right)\right) \\
& \left.\left.\cdot\left(\left(0.37+0.02 H_{0} t_{0}\right)\left(-0.054 H_{0}+\left(-\frac{0.14}{H_{0}}+t_{0}\right)\left(1+0.054 H_{0} t_{0}\right)\right)^{2}\right)^{-1}\right)\right),
\end{aligned}
$$

where $\Gamma(a, z)=\int_{z}^{\infty} e^{-t} t^{a-1} d t$

From the above limits, one can see that the EoS parameter is determined by the viscosity of the EHRDE and does not blow up in the past or future. This observation is consistent with [43], where the RDE was considered with barotropic fluid and the EoS parameter was seen not to blow up in the past of the future. In Figure 1 we have plotted the EoS parameter and observed that $w_{\mathrm{DE}}<-1$; that is, the phantom phase is attained. It is noted that the phantom barrier $w_{\mathrm{DE}}=-1$ is never being crossed and in the very late stage $w_{\mathrm{DE}} \ll-1$. In Figure 2, the effect of bulk viscosity on the thermodynamic pressure is visualized. We observe that $p+\Pi \ll 0$ during the evolution. Figure 3 shows that $\dot{\Pi}>0$ and from this we can understand that the effect of bulk viscosity on the thermodynamic pressure is increasing with evolution of the universe. However, $\left|p_{\text {eff }}\right|$ is gradually decreasing with evolution of the universe. Figure 4 shows that the bulk viscosity coefficient $\xi$ is a monotone increasing function of cosmic time.

2.2. Israel-Stewart Approach for $a(t)=\left(t-t_{0}\right)^{\beta /(1-\alpha)}$. In Section 2.1, instead of making any assumption on scale factor, we have derived solution for scale factor in (12). In the present section the scale factor is chosen as

$$
a(t)=a_{0}\left(t-t_{0}\right)^{\beta /(1-\alpha)}
$$

This leads to

$$
\begin{aligned}
& H=\frac{\beta}{\left(t-t_{0}\right)(1-\alpha)}, \\
& \dot{H}=-\frac{\beta}{\left(t-t_{0}\right)^{2}(1-\alpha)} .
\end{aligned}
$$

Based on (28), the reconstructed density of EHRDE is

$$
\rho_{\mathrm{DE}}=\frac{3 \beta^{2}(2 \alpha-1)}{\left(t-t_{0}\right)^{2}(1-\alpha)^{2}}
$$

For $\rho>0$, one needs $\alpha>1 / 2$. Accordingly the bulk viscosity coefficient and relaxation times (18) get reconstructed and hence evolution equation for $\Pi$ in the framework of the truncated Israel-Stewart theory given by (19) is solved to get

$$
\begin{aligned}
\Pi & =C_{2} e^{-\left(t-t_{0}\right)^{3}(-1+\alpha)^{2} / 9(-1+2 \alpha) \beta^{2} \eta} \\
& +e^{-\left(t-t_{0}\right)^{3}(-1+\alpha)^{2} / 9(-1+2 \alpha) \beta^{2} \eta}(-1+2 \alpha) \\
& \cdot \beta^{3}\left(-9 e^{\left(t-t_{0}\right)^{3}(-1+\alpha)^{2} / 9(-1+2 \alpha) \beta^{2} \eta}\right. \\
& +3^{2 / 3}\left(-\frac{\left(t-t_{0}\right)^{3}(-1+\alpha)^{2}}{(-1+2 \alpha) \beta^{2} \eta}\right)^{2 / 3} \\
& \left.\cdot \Gamma\left[\frac{1}{3},-\frac{\left(t-t_{0}\right)^{3}(-1+\alpha)^{2}}{9(-1+2 \alpha) \beta^{2} \eta}\right]\right)\left(2\left(t-t_{0}\right)^{2}\right. \\
& \left.\cdot(-1+\alpha)^{3}\right)^{-1},
\end{aligned}
$$

and hence

$$
\begin{aligned}
\dot{\Pi} & =\frac{e^{-\left(t-t_{0}\right)^{3}(-1+\alpha)^{2} / 9(-1+2 \alpha) \beta^{2} \eta}}{6\left(t-t_{0}\right)^{3}(-1+\alpha)^{3}(-1+2 \alpha) \beta^{2} \eta^{2}}\left(\eta \left(-2 C_{2}(t\right.\right. \\
& \left.-t_{0}\right)^{5}(-1+\alpha)^{5}+9 e^{\left(t-t_{0}\right)^{3}(-1+\alpha)^{2} / 9(-1+2 \alpha) \beta^{2} \eta}(-1 \\
& \left.+2 \alpha) \beta^{3}\left(\left(t-t_{0}\right)^{3}(-1+\alpha)^{2}+6(-1+2 \alpha) \beta^{2} \eta\right)\right) \\
& \left.+\left(t-t_{0}\right)^{6}(-1+\alpha)^{4} \beta E_{2 / 3}[T]\right),
\end{aligned}
$$

where $T=-\left(t-t_{0}\right)^{3}(-1+\alpha)^{2} / 9(-1+2 \alpha) \beta^{2} \eta$. Subsequently, $p_{\mathrm{DE}}$ becomes

$$
\begin{aligned}
p_{\mathrm{DE}} & =C_{2} e^{-\left(t-t_{0}\right)^{3}(-1+\alpha)^{2} / 9(-1+2 \alpha) \beta^{2} \eta} \\
& -\frac{2 \beta}{\left(t-t_{0}\right)^{2}(-1+\alpha)}-\frac{3 \beta^{2}}{\left(t-t_{0}\right)^{2}(-1+\alpha)^{2}} \\
& +e^{-\left(t-t_{0}\right)^{3}(-1+\alpha)^{2} / 9(-1+2 \alpha) \beta^{2} \eta}(-1+2 \alpha)
\end{aligned}
$$




$$
\begin{aligned}
& \cdot \beta^{3}\left(9 e^{\left(t-t_{0}\right)^{3}(-1+\alpha)^{2} / 9(-1+2 \alpha) \beta^{2} \eta}\right. \\
& -3^{2 / 3}\left(-\frac{\left(t-t_{0}\right)^{3}(-1+\alpha)^{2}}{(-1+2 \alpha) \beta^{2} \eta}\right)^{2 / 3} \\
& \left.\cdot \Gamma\left[\frac{1}{3},-\frac{\left(t-t_{0}\right)^{3}(-1+\alpha)^{2}}{9(-1+2 \alpha) \beta^{2} \eta}\right]\right)\left(2\left(t-t_{0}\right)^{2}\right. \\
& \left.\cdot(-1+\alpha)^{3}\right)^{-1},
\end{aligned}
$$

and hence EoS parameter $w_{\mathrm{DE}}$ is

$$
\begin{aligned}
& w_{\mathrm{DE}} \\
& =\frac{\left(t-t_{0}\right)^{2}(-1+\alpha)^{2}}{3(-1+2 \alpha) \beta^{2}}\left(-C_{2} e^{-\left(t-t_{0}\right)^{3}(-1+\alpha)^{2} / 9(-1+2 \alpha) \beta^{2} \eta}\right. \\
& -\frac{2 \beta}{\left(t-t_{0}\right)^{2}(-1+\alpha)}-\frac{3 \beta^{2}}{\left(t-t_{0}\right)^{2}(-1+\alpha)^{2}} \\
& +e^{-\left(t-t_{0}\right)^{3}(-1+\alpha)^{2} / 9(-1+2 \alpha) \beta^{2} \eta}(-1+2 \alpha) \\
& \cdot \beta^{3}\left(9 e^{\left(t-t_{0}\right)^{3}(-1+\alpha)^{2} / 9(-1+2 \alpha) \beta^{2} \eta}\right. \\
& -3^{2 / 3}\left(-\frac{\left(t-t_{0}\right)^{3}(-1+\alpha)^{2}}{(-1+2 \alpha) \beta^{2} \eta}\right)^{2 / 3} \\
& \left.\cdot \Gamma\left[\frac{1}{3},-\frac{\left(t-t_{0}\right)^{3}(-1+\alpha)^{2}}{9(-1+2 \alpha) \beta^{2} \eta}\right]\right)\left(2\left(t-t_{0}\right)^{2}\right. \\
& \left.\left.\cdot(-1+\alpha)^{3}\right)^{-1}\right) \cdot
\end{aligned}
$$

In the above equations, $E_{n}[z]=\int_{1}^{\infty}\left(e^{-z t} / t^{n}\right) d t$.

In Figures 5, 6, and 7, the red, green, and blue lines correspond to $\eta=0.00009,0.0002$, and 0.00015 , respectively. In all the cases in this section, $\alpha=0.9180$ and $\beta=0.3701$. In Figure 5 we plot $w_{\mathrm{DE}}$ and observe that the EoS parameter shows a clear transition from $w_{\mathrm{DE}}>-1$ to $w_{\mathrm{DE}}<-1$, that is, from quintessence to phantom. Hence, the model behaves like "Quintom." This is in contradiction with what happened in the model without any specific choice of scale factor, where $w_{\mathrm{DE}}<-1$ that implies "phantom" behavior of the EoS parameter. Similar to the earlier case, the effective pressure (Figure 6) is decaying with the evolution of the universe. However, time derivative of the bulk viscous pressure $\dot{\Pi}$ stays at positive level (Figure 7). This indicates that the effect of bulk viscous pressure increases with time. This means that the negative bulk viscous pressure gives a significant contribution to the total negative pressure of the EHRDE. The positive time derivative of bulk viscous pressure and gradually decaying

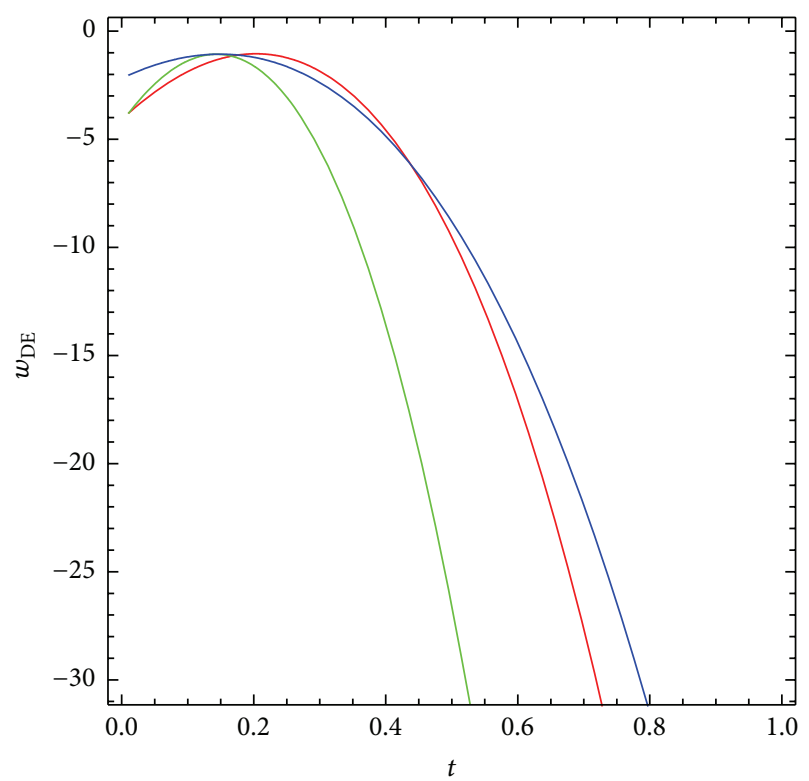

FIGURE 1: EoS parameter based on (22). Red, green, and blue lines correspond to $\{\alpha, \beta\}$ combination of $\{0.9735,0.3701\}$ with $\eta=3.5 \times$ $10^{-4}, 3.0 \times 10^{-4}$, and $2.5 \times 10^{-4}$, respectively.

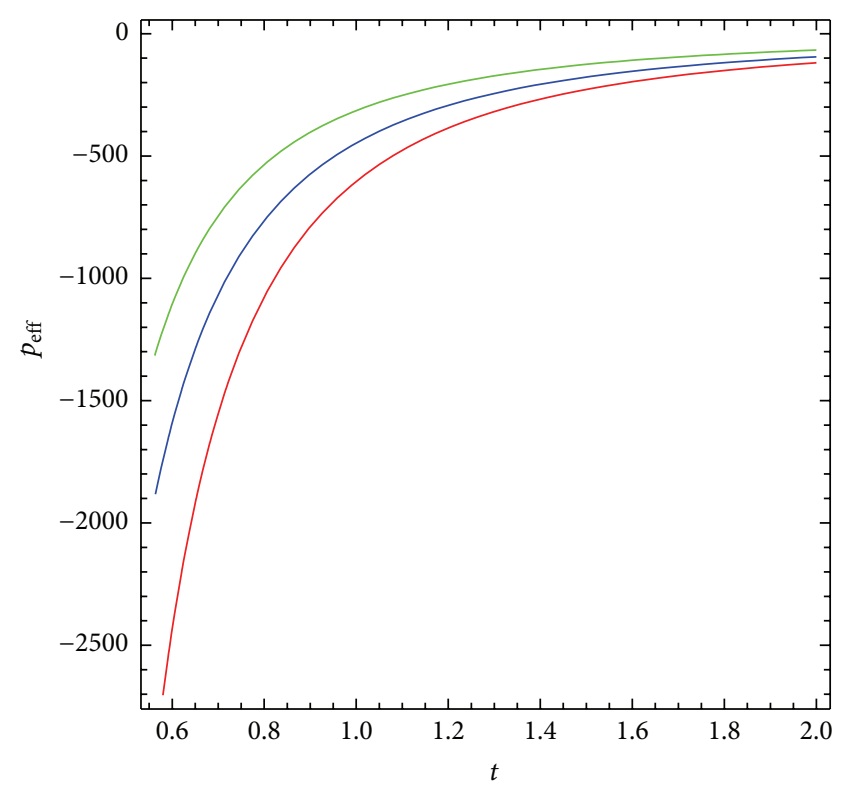

FIgURE 2: Plot of $p_{\text {eff }}=p+\Pi$. Red, green, and blue lines correspond to $\{\alpha, \beta\}$ combination of $\{0.9735,3701\}$ and $\{0.9735,3701\}$ with $\eta=$ $3.5 \times 10^{-4}, 3.0 \times 10^{-4}$, and $2.5 \times 10^{-4}$, respectively.

effective pressure indicates that nonequilibrium bulk viscous pressure is small compared to the local equilibrium pressure.

\section{State-Finder Parameters}

3.1. Model without Any Specific Choice of Scale Factor. Sahni et al. [56] and Alam et al. [57] introduced a pair of cosmological 


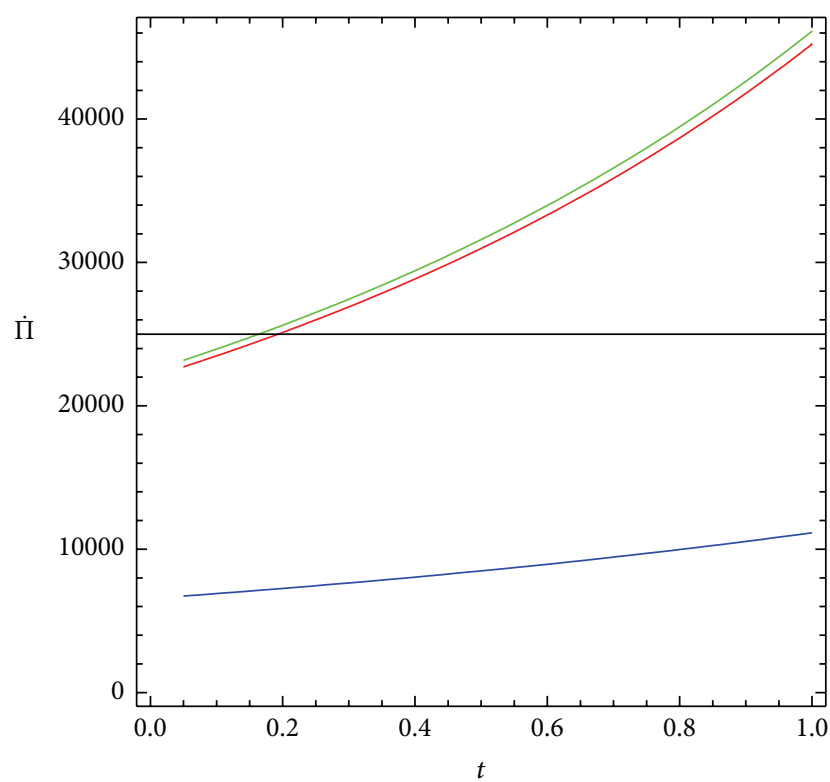

Figure 3: Plot of $\dot{\Pi}$ based on (20). Red, green, and blue lines correspond to $\{\alpha, \beta\}$ combination of $\{0.9735,3701\}$ with $\eta=3.5 \times$ $10^{-4}, 3.0 \times 10^{-4}$, and $2.5 \times 10^{-4}$, respectively.

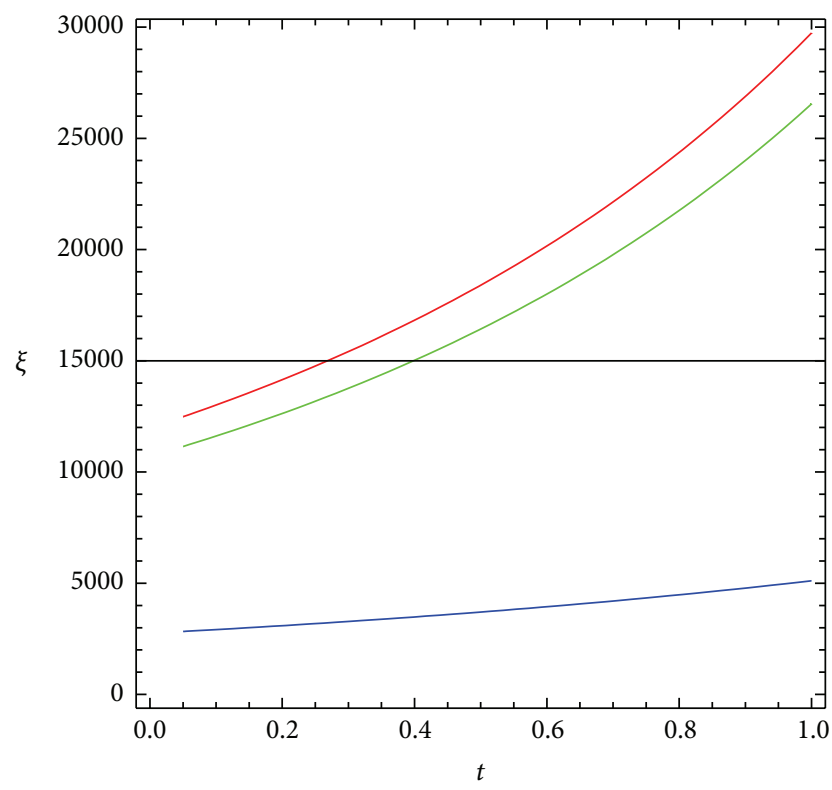

FIGURE 4: Plot of the bulk viscosity coefficient $\xi$. Red, green, and blue lines correspond to $\{\alpha, \beta\}$ combination of $\{0.9735,3701\}$ with $\eta=3.5 \times 10^{-4}, 3.0 \times 10^{-4}$, and $2.5 \times 10^{-4}$, respectively.

parameters $\{r, s\}$ (the so-called "state-finder parameters") that seem to be promising candidates for the purpose of discrimination between the various contenders of dark energy. If the $\{r-s\}$ trajectory meets the point $\{r=1, s=0\}$, then the model is said to attain $\Lambda \mathrm{CDM}$ phase of the universe. In the literature, quite a good number of works are available,

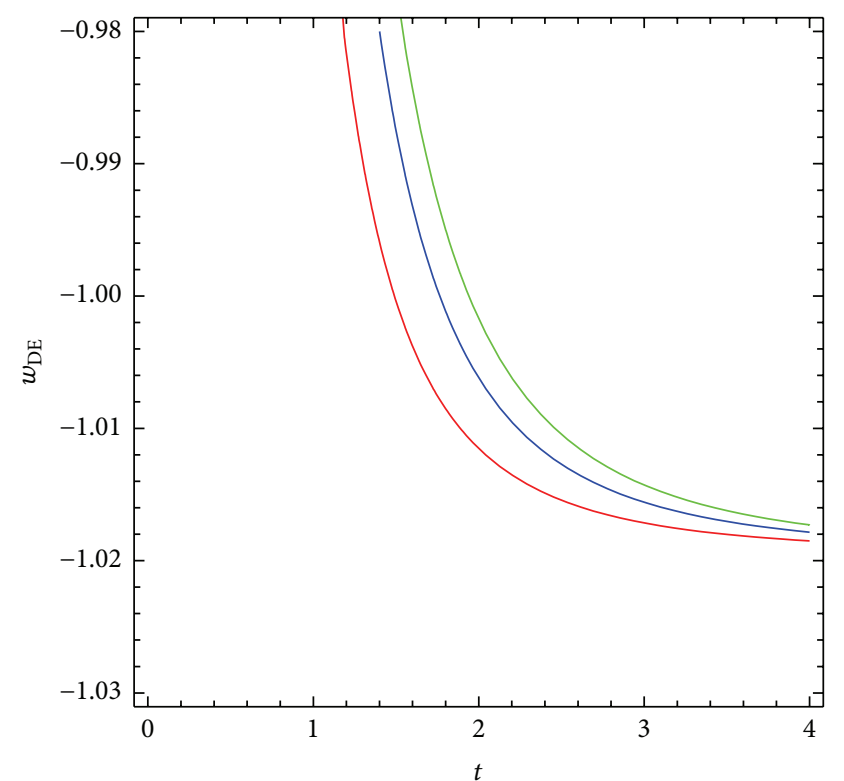

Figure 5: Plot of $w_{\text {DE }}$ for scale factor in (33).

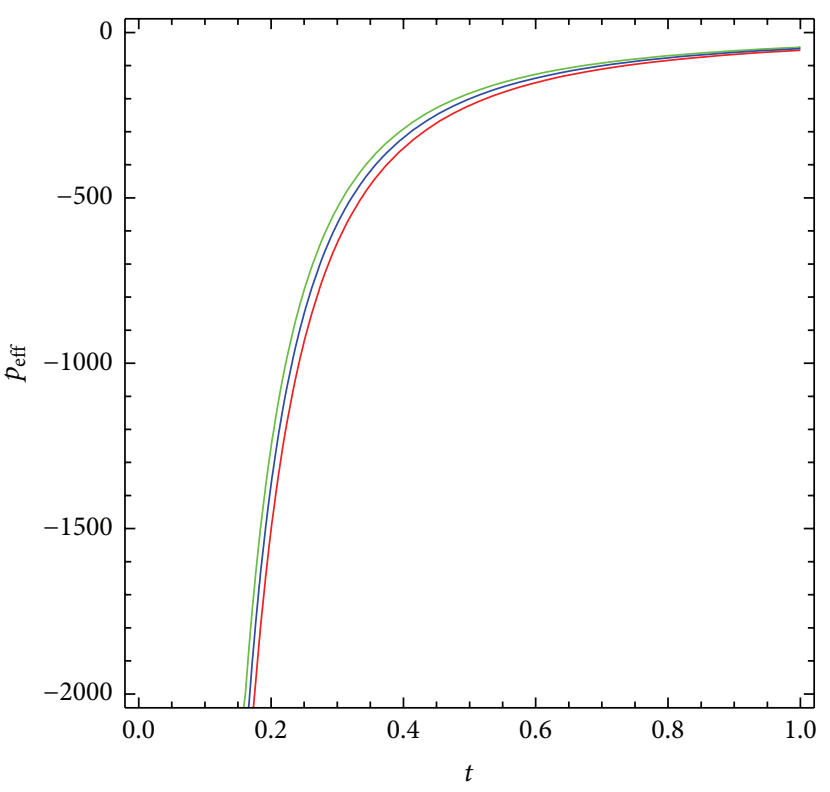

FIgURE 6: Effective pressure $p_{\text {eff }}=p_{\mathrm{DE}}+\Pi$ based on (30) and (32).

where dark energy models have been explored through statefinder trajectories, for example, [58-60]. The state-finder parameters are given by

$$
\begin{aligned}
& r=q+2 q^{2}+\frac{\dot{q}}{H}, \\
& s=\frac{r-1}{3(q-1 / 2)} .
\end{aligned}
$$

In (34), deceleration parameter $q$ is given by

$$
q=-\frac{\ddot{a} a}{\dot{a}^{2}},
$$




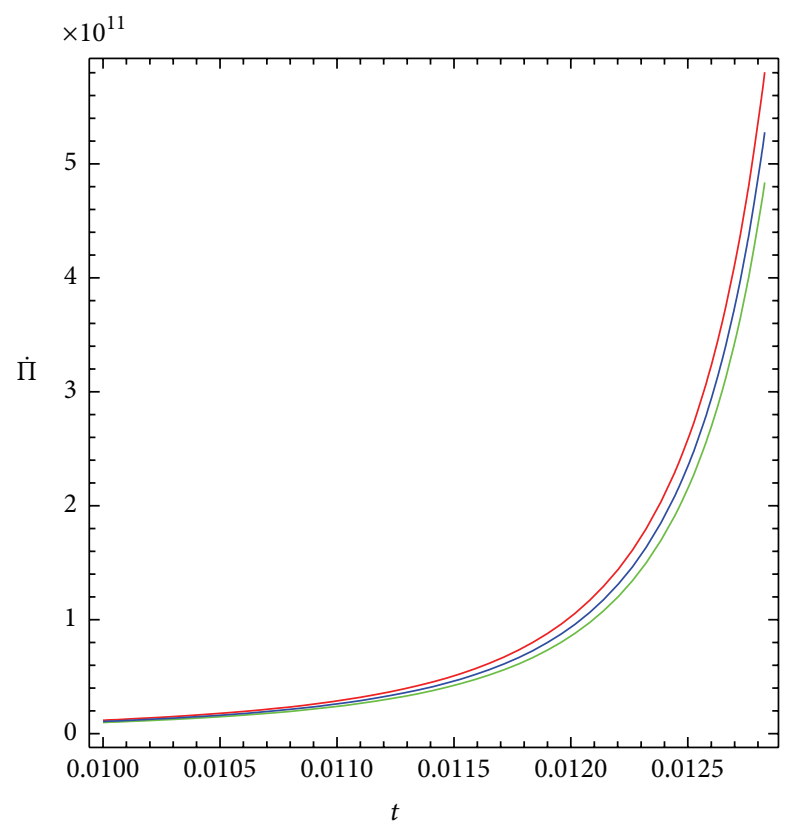

FIgURE 7: Time evolution of bulk viscous pressure $\dot{\Pi}$ based on (31).

where $a$ is the scale factor as available in (12). Hence, in the current framework, (34) take the form

$$
\begin{aligned}
r & =\frac{H_{0}^{2}(\alpha-1)^{2}(-2+\beta /(\alpha-1))(-1+\beta /(\alpha-1))}{\left(H_{0}\left(t-t_{0}\right)(\alpha-1)+\beta\right)^{2}} \\
s & =2\left(-1+\frac{H_{0}^{2}(\alpha-1-\beta)(-2+2 \alpha-\beta)}{\left(H_{0}\left(t-t_{0}\right)(\alpha-1)+\beta\right)^{2}}\right) \\
& \cdot\left(3 \left(-3-\frac{2}{\beta}+\frac{2 \alpha}{\beta}\right.\right. \\
& +\left(C_{1} e^{-\left(H_{0}\left(-1+t t_{0}\right)(\alpha-1)-t \beta\right)^{3} / 9 H_{0}^{2}\left(H_{0} t_{0}(\alpha-1)-\beta\right) \beta^{2} \eta}\right. \\
& \cdot\left(H_{0}\left(t-t_{0}\right)(\alpha-1)+\beta\right)^{2} \\
& \cdot\left(2 H_{0}^{2}\left(1-4 t t_{0}+3 t^{2} t_{0}^{2}\right)(\alpha-1)^{2} \beta\right. \\
& -2 H_{0} t\left(-2+3 t t_{0}\right)(\alpha-1) \beta^{2}+2 t^{2} \beta^{3} \\
& +H_{0}^{3}\left(-2 t_{0}(\alpha-1)^{3}+4 t t_{0}^{2}(\alpha-1)^{3}\right. \\
& -2 t^{2} t_{0}^{3}(\alpha-1)^{3}+9 \\
& \left.\cdot e^{\left(H_{0}\left(-1+t t_{0}\right)(\alpha-1)-t \beta\right)^{3} / 9 H_{0}^{2}\left(H_{0} t_{0}(\alpha-1)-\beta\right) \beta^{2} \eta} \beta^{3}\right) \\
& -3^{2 / 3} H_{0}^{3} \beta^{3}\left(-\frac{\left(H_{0}\left(-1+t t_{0}\right)(\alpha-1)-t \beta\right)^{3}}{H_{0}^{2}\left(H_{0} t_{0}(\alpha-1)-\beta\right) \beta^{2} \eta}\right)^{2 / 3}
\end{aligned}
$$

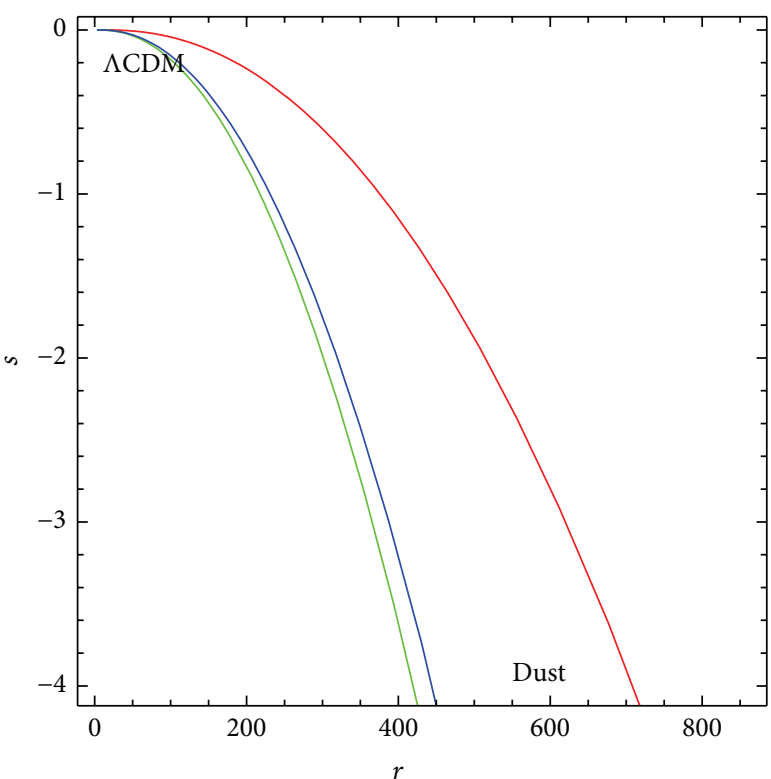

FIGURE 8: The $\{r-s\}$ trajectory based on (36). Red, green, and blue lines correspond to $\{\alpha, \beta\}$ combination of $\{0.9735,3701\}$ with $\eta=$ $3.5 \times 10^{-4}, 3.0 \times 10^{-4}$, and $2.5 \times 10^{-4}$, respectively.

$$
\begin{aligned}
& \left.\left.\cdot \Gamma\left[\frac{1}{3},-\frac{\left(H_{0}\left(-1+t t_{0}\right)(\alpha-1)-t \beta\right)^{3}}{9 H_{0}^{2}\left(H_{0} t_{0}(\alpha-1)-\beta\right) \beta^{2} \eta}\right]\right)\right) \\
& \cdot\left(2 H_{0}^{2}\left(H_{0} t_{0}(\alpha-1)-\beta\right) \beta^{2}\right. \\
& \left.\left.\left.\cdot\left(H_{0}\left(-1+t t_{0}\right)(\alpha-1)-t \beta\right)^{2}\right)^{-1}\right)\right)^{-1} \cdot
\end{aligned}
$$

In Figure 8, we observe that the $\{r-s\}$ trajectory can attain the $\Lambda \mathrm{CDM}$ point; that is, $\{r=1, s=0\}$. Again, for finite $r$, we observe that $s \rightarrow-\infty$ that corresponds to dust phase. Thus, we may conclude that the viscous EHRDE interpolates between dust and $\Lambda \mathrm{CDM}$ phases of the universe. However, the model deviates significantly from $\Lambda \mathrm{CDM}$.

3.2. Model with Scale Factor $a(t)=\left(t-t_{0}\right)^{\beta /(1-\alpha)}$. For the choice of scale factor $a(t)=\left(t-t_{0}\right)^{\beta /(1-\alpha)}$, we get from (34) the state-finder parameters as

$$
\begin{aligned}
r= & \frac{1}{2}\left(1+e^{-\left(t-t_{0}\right)^{3}(-1+\alpha)^{2} / 9(-1+2 \alpha) \beta^{2} \eta}\right. \\
& \cdot\left(-2 C_{2}\left(t-t_{0}\right)^{2}(-1+\alpha)^{3} \eta+e^{\left(t-t_{0}\right)^{3}(-1+\alpha)^{2} / 9(-1+2 \alpha) \beta^{2} \eta} \beta\right. \\
& \cdot\left(-4(-1+\alpha)^{2}-6(-1+\alpha) \beta+9(-1+2 \alpha) \beta^{2}\right) \eta \\
& \left.+\left(t-t_{0}\right)^{3}(-1+\alpha)^{2} \beta E_{2 / 3}[T]\right)\left(2(1+\alpha(-3+2 \alpha)) \beta^{2} \eta\right)^{-1} \\
& -\frac{1}{6(1+\alpha(-3+2 \alpha)) \beta^{5} \eta^{2}} e^{-\left(t-t_{0}\right)^{3}(-1+\alpha)^{2} / 9(-1+2 \alpha) \beta^{2} \eta}\left(t-t_{0}\right)^{2} \\
& \cdot(-1+\alpha)^{3}\left(\beta ^ { 2 } \eta \left(e^{\left(t-t_{0}\right)^{3}(-1+\alpha)^{2} / 9(-1+2 \alpha) \beta^{2} \eta}\left(t-t_{0}\right)\right.\right.
\end{aligned}
$$




$$
\begin{aligned}
& \cdot\left(-4(-1+\alpha)^{2}-6(-1+\alpha) \beta+9(-1+2 \alpha) \beta^{2}\right) \\
& \cdot((-1+2 \alpha) \beta)^{-1}-12 C_{2}(-1+\alpha) \eta \\
& \left.+\frac{\left(t-t_{0}\right)^{4}(-1+\alpha)^{2} E_{-1 / 3}[T]}{(-1+2 \alpha) \beta \eta}+9\left(t-t_{0}\right) \beta E_{2 / 3}[T]\right) \\
& -\left(t-t_{0}\right)\left(-2 C_{2}\left(t-t_{0}\right)^{2}(-1+\alpha)^{3} \eta\right. \\
& +e^{\left(t-t_{0}\right)^{3}(-1+\alpha)^{2} / 9(-1+2 \alpha) \beta^{2} \eta} \beta \\
& \cdot\left(-4(-1+\alpha)^{2}-6(-1+\alpha) \beta+9(-1+2 \alpha) \beta^{2}\right) \eta \\
& \left.+\left(t-t_{0}\right)^{3}(-1+\alpha)^{2} \beta E_{2 / 3}[T]\right) \\
& \left.\cdot(-1+2 \alpha)^{-1}\right)+\left(1+e^{-\left(t-t_{0}\right)^{3}(-1+\alpha)^{2} / 9(-1+2 \alpha) \beta^{2} \eta}\right. \\
& \cdot\left(-2 C_{2}\left(t-t_{0}\right)^{2}(-1+\alpha)^{3} \eta+e^{\left(t-t_{0}\right)^{3}(-1+\alpha)^{2} / 9(-1+2 \alpha) \beta^{2} \eta} \beta\right. \\
& \cdot\left(-4(-1+\alpha)^{2}-6(-1+\alpha) \beta+9(-1+2 \alpha) \beta^{2}\right) \eta \\
& \left.+\left(t-t_{0}\right)^{3}(-1+\alpha)^{2} \beta E_{2 / 3}[T]\right) \\
& \left.\left.\cdot\left(2(1+\alpha(-3+2 \alpha)) \beta^{2} \eta\right)^{-1}\right)^{2}\right), \\
& s=\left(2 e^{\left(t-t_{0}\right)^{3}(-1+\alpha)^{2} / 9(-1+2 \alpha) \beta^{2} \eta}(1+\alpha(-3+2 \alpha)) \beta^{2} \eta\right. \\
& \cdot\left(-1+e^{-\left(t-t_{0}\right)^{3}(-1+\alpha)^{2} / 9(-1+2 \alpha) \beta^{2} \eta}\right. \\
& \text { - }\left(-2 C_{2}\left(t-t_{0}\right)^{2}(-1+\alpha)^{3} \eta\right. \\
& +e^{\left(t-t_{0}\right)^{3}(-1+\alpha)^{2} / 9(-1+2 \alpha) \beta^{2} \eta} \beta \\
& \cdot\left(-4(-1+\alpha)^{2}-6(-1+\alpha) \beta+9(-1+2 \alpha) \beta^{2}\right) \eta \\
& \left.+\left(t-t_{0}\right)^{3}(-1+\alpha)^{2} \beta E_{2 / 3}[T]\right) \\
& \cdot\left(2(1+\alpha(-3+2 \alpha)) \beta^{2} \eta\right)^{-1} \\
& -\frac{e^{-\left(t-t_{0}\right)^{3}(-1+\alpha)^{2} / 9(-1+2 \alpha) \beta^{2} \eta}}{6(1+\alpha(-3+2 \alpha)) \beta^{5} \eta^{2}}\left(t-t_{0}\right)^{2}(-1+\alpha)^{3} \\
& \cdot\left(\beta ^ { 2 } \eta \left(e^{\left(t-t_{0}\right)^{3}(-1+\alpha)^{2} / 9(-1+2 \alpha) \beta^{2} \eta}\left(t-t_{0}\right)\right.\right. \\
& \cdot\left(-4(-1+\alpha)^{2}-6(-1+\alpha) \beta+9(-1+2 \alpha) \beta^{2}\right) \\
& \cdot((-1+2 \alpha) \beta)^{-1}-12 C_{2}(-1+\alpha) \eta \\
& \left.+\frac{\left(t-t_{0}\right)^{4}(-1+\alpha)^{2} E_{-1 / 3}[T]}{(-1+2 \alpha) \beta \eta}+9\left(t-t_{0}\right) \beta E_{2 / 3}[T]\right) \\
& -\left(t-t_{0}\right)\left(-2 C_{2}\left(t-t_{0}\right)^{2}(-1+\alpha)^{3} \eta\right. \\
& +e^{\left(t-t_{0}\right)^{3}(-1+\alpha)^{2} / 9(-1+2 \alpha) \beta^{2} \eta} \beta \\
& \cdot\left(-4(-1+\alpha)^{2}-6(-1+\alpha) \beta+9(-1+2 \alpha) \beta^{2}\right) \eta
\end{aligned}
$$

$$
\begin{aligned}
& \left.\left.+\left(t-t_{0}\right)^{3}(-1+\alpha)^{2} \beta E_{2 / 3}[T]\right)(-1+2 \alpha)^{-1}\right) \\
& +\left(1+e^{-\left(t-t_{0}\right)^{3}(-1+\alpha)^{2} / 9(-1+2 \alpha) \beta^{2} \eta}\right. \\
& \cdot\left(-2 C_{2}\left(t-t_{0}\right)^{2}(-1+\alpha)^{3} \eta+e^{\left(t-t_{0}\right)^{3}(-1+\alpha)^{2} / 9(-1+2 \alpha) \beta^{2} \eta} \beta\right. \\
& \cdot\left(-4(-1+\alpha)^{2}-6(-1+\alpha) \beta+9(-1+2 \alpha) \beta^{2}\right) \eta \\
& \left.+\left(t-t_{0}\right)^{3}(-1+\alpha)^{2} \beta E_{2 / 3}[T]\right) \\
& \left.\left.\left.\cdot\left(2(1+\alpha(-3+2 \alpha)) \beta^{2} \eta\right)^{-1}\right)^{2}\right)\right) \\
& \cdot\left(3 \left(-2 C_{2}\left(t-t_{0}\right)^{2}(-1+\alpha)^{3} \eta\right.\right. \\
& +e^{\left(t-t_{0}\right)^{3}(-1+\alpha)^{2} / 9(-1+2 \alpha) \beta^{2} \eta} \beta \\
& \cdot\left(-4(-1+\alpha)^{2}-6(-1+\alpha) \beta+9(-1+2 \alpha) \beta^{2}\right) \eta \\
& \left.\left.+\left(t-t_{0}\right)^{3}(-1+\alpha)^{2} \beta E_{2 / 3}[T]\right)\right)^{-1} .
\end{aligned}
$$

In Figure 9, we have plotted the state-finder trajectories for the model with scale factor $a(t)=\left(t-t_{0}\right)^{\beta /(1-\alpha)}$. It is observed that the fixed $\Lambda$ CDM point $\{r=1, s=0\}$ is not attained by the trajectories. However, for finite $r$, we observe $s \rightarrow-\infty$ that corresponds to the dust phase of the universe.

\section{Generalized Second Law of Thermodynamics}

Discovery of black hole thermodynamics in the 70s [61-63] prompted physicists to study the thermodynamics of cosmological models of the universe. Semiclassical description in black hole physics shows that a black hole behaves like a black 
body that is emitting thermal radiation with temperature and entropy. This temperature and the entropy are known as Hawking temperature and Bekenstein entropy, respectively, [64-66]. This entropy is proportional to surface area $A$ of black hole, which, according to Hawkings area theorem, cannot decrease. Based on the conjectured proportionality between entropy and horizon area of black hole, a generalized version of the second law of thermodynamics was proposed by Bekenstein. According to the proposal of Bekenstein, the sum of black hole entropy and the entropy of matter and radiation in the region exterior to black hole cannot decrease. The GSL provides a relation between gravitation, thermodynamics, and quantum theory. In a very recent work [67] conjectured a novel GSL that can be applied in cosmology irrespective of the presence of event horizon.

Reference [42] examined the validity of the generalized GSL in a nonflat universe in the presence of viscous dark energy. Setare [68] investigated the validity of the generalized second law of thermodynamics for the Quintom model of dark energy. Considering the universe as a closed bounded system filled with $n$ component fluids, Bamba et al. [69] studied the generalized second law in $f(T)$ cosmology. Reference [70] investigated the validity of the generalized second law in the context of interacting $f(R)$ gravity. We consider the universe. To check the generalized second law of thermodynamics, we have to examine the evolution of the total entropy $S_{A}+S_{\mathrm{DE}}$, where $S_{A}$ denotes the entropy of the apparent horizon and $S_{\mathrm{DE}}$ denotes the entropy of the fluid inside the horizon. For the FRW universe, the apparent horizon radius reads [42]

$$
\widetilde{r}_{A}=\frac{1}{\sqrt{H^{2}+k / a^{2}}} .
$$

In a flat universe $k=0$ and (39) becomes

$$
\tilde{r}_{A}=\frac{1}{H} \text {. }
$$

Temperature on the apparent horizon is defined as [42]

$$
T_{A}=\frac{1}{2 \pi \widetilde{r}_{A}}\left(1-\frac{\dot{\tilde{r}}_{A}}{2 H \tilde{r}_{A}}\right) .
$$

The entropy associated with the apparent horizon is [42]

$$
S_{A}=\frac{A}{4 G}=\frac{\pi \tilde{r}_{A}^{2}}{G},
$$

where $A=4 \pi \widetilde{r}_{A}^{2}$ is the area of the apparent horizon. It has been shown by some calculations in [42] that for viscous dark energy dominated flat universe enveloped by the apparent horizon

$$
\begin{aligned}
& T_{A} \dot{S}_{A} \\
& \quad=4 \pi H \widetilde{r}_{A}^{3}\left(\rho_{\mathrm{DE}}\left(1+w_{\mathrm{DE}}\right)-3 H \xi\right)\left(1-\frac{\dot{\widetilde{r}}_{A}}{2 H \widetilde{r}_{A}}\right) .
\end{aligned}
$$

In the present case, the above can be rewritten as

$$
\begin{aligned}
& T_{A} \dot{S}_{A} \\
& \quad=4 \pi H \widetilde{r}_{A}^{3}\left(\rho_{\mathrm{DE}}\left(1+w_{\mathrm{DE}}\right)+\tau \dot{\Pi}+\Pi\right)\left(1-\frac{\dot{\vec{r}}_{A}}{2 H \widetilde{r}_{A}}\right) .
\end{aligned}
$$

The entropy of the viscous dark energy inside the apparent horizon, $S_{\mathrm{DE}}$, can be related to its energy $E_{\mathrm{D}}=\rho_{\mathrm{D}} V$ and its pressure as

$$
\begin{aligned}
T_{\mathrm{D}} \dot{S}_{\mathrm{D}} & =V \dot{\rho}_{\mathrm{DE}}+\left(\rho_{\mathrm{DE}}\left(1+w_{\mathrm{DE}}\right)-3 H \xi\right) \dot{V} \\
& =V \dot{\rho}_{\mathrm{DE}}+\left(\rho_{\mathrm{DE}}\left(1+w_{\mathrm{DE}}\right)+\tau \dot{\Pi}+\Pi\right) \dot{V}
\end{aligned}
$$

where $T_{\mathrm{DE}}$ is the temperature of the viscous dark energy and $V=(4 / 3) \pi \widetilde{r}_{A}^{3}$ is the volume enveloped by the apparent horizon. Under the assumption that the thermal system bounded by the apparent horizon remains in equilibrium, that is, temperature of the system is uniform and the temperature on the horizon is equal to temperature of the fluid inside the horizon, we have $T_{A}=T_{\mathrm{DE}}=T$. It has been shown in [42] by some simple calculation that

$$
T\left(\dot{S}_{A}+\dot{S}_{\mathrm{DE}}\right)=\frac{A}{2}\left(\rho_{\mathrm{DE}}\left(1+w_{\mathrm{DE}}\right)+\tau \dot{\Pi}+\Pi\right) \dot{\widetilde{r}}_{A}
$$

Using (14), (15), and (20) in (46), we get

$$
\begin{aligned}
& \dot{S}_{A}+ \dot{S}_{\mathrm{DE}}=\frac{2 \pi}{H_{0} \beta^{3}}(-1+\alpha)\left(H_{0}\left(t-t_{0}\right)(-1+\alpha)+\beta\right) \\
& \cdot\left(\frac{\beta}{G}-\frac{8 \pi}{\alpha-2 \beta-1}\left(-2 \beta^{2}+\frac{1}{H_{0}^{2}}\left(H_{0}\left(t-t_{0}\right)(-1+\alpha)+\beta\right)^{2}\right.\right. \\
& \cdot\left(C_{1} e^{-\chi}-\frac{e^{-\chi} H_{0}^{3} \beta^{3}\left(9 e^{\chi}-3^{2 / 3}(-\chi)^{2 / 3} \Gamma[1 / 3,-\chi]\right)}{2\left(H_{0} t_{0}(-1+\alpha)-\beta\right)\left(H_{0}\left(-1+t t_{0}\right)(-1+\alpha)-t \beta\right)^{2}}\right. \\
&+ e^{-\chi} \beta^{2} \eta\left(-\frac{2 C_{1}\left(H_{0}\left(-1+t t_{0}\right)(-1+\alpha)-t \beta\right)^{2}}{\beta^{2} \eta}\right. \\
&- \frac{63^{2 / 3} H_{0}^{3} \beta \Gamma[1 / 3,-\chi]}{\left(H_{0} t_{0}(-1+\alpha)-\beta\right) \eta(-\chi)^{1 / 3}} \\
&+ \frac{6 H_{0}^{5} \beta^{3}\left(9 e^{\chi}-3^{2 / 3}(-\chi)^{2 / 3} \Gamma[1 / 3,-\chi]\right)}{\left(H_{0}\left(-1+t t_{0}\right)(-1+\alpha)-t \beta\right)^{3}} \\
&+\left.+\frac{H_{0}^{3} \beta\left(9 e^{\chi}-3^{2 / 3}(-\chi)^{2 / 3} \Gamma[1 / 3,-\chi]\right)}{\left(H_{0} t_{0}(-1+\alpha)-\beta\right) \eta}\right) \\
&+\left(2 \left(H_{0}\left(t-H_{0}^{3} \beta^{3}(-\chi)^{2 / 3} \Gamma\left[\frac{1}{3},-\chi\right]\right)\right.\right. \\
&+ H_{0}^{2}\left(2 \beta(\alpha-1)-e^{-\chi}\left(H_{0}\left(t-t_{0}\right)(-1+\alpha)+\beta\right)^{2}\right. \\
&+\left(-9 e^{\chi} H_{0}^{3} \beta^{3}+2 C_{1}\left(H_{0} t_{0}(-1+\alpha)-\beta\right)\right. \\
&+(-1+\alpha)-t \beta)^{2} \\
&+1
\end{aligned}
$$




$$
\begin{aligned}
& \left(2 H_{0}^{2}\left(H_{0} t_{0}(-1+\alpha)-\beta\right)\right. \\
& \left.\left.\cdot\left(H_{0}\left(-1+t t_{0}\right)(-1+\alpha)-t \beta\right)^{2}\right)^{-1}\right) \\
& \left.\left.\left.\cdot\left(\left(H_{0}\left(t-t_{0}\right)(-1+\alpha)+\beta\right)^{2}\right)^{-1}\right)\right)\right),
\end{aligned}
$$

where $\chi=\left(H_{0}\left(-1+t t_{0}\right)(-1+\alpha)-t \beta\right)^{3} / 9 H_{0}^{2}\left(H_{0} t_{0}(-1+\right.$ $\alpha)-\beta) \beta^{2} \eta$. In Figure 10, we have plotted $T\left(\dot{S}_{A}+\dot{S}_{\mathrm{DE}}\right)$ based on (47) with cosmic time $t$ and $\eta$ ranging from 0.0002 to 0.0005. We have observed that $T\left(\dot{S}_{A}+\dot{S}_{\mathrm{DE}}\right)$ is staying at positive level. Since $T>0$, Figure 10 indicates that $\dot{S}_{A}+$ $\dot{S}_{\mathrm{DE}} \geq 0$. This indicates validity of the generalized second law of thermodynamics in a universe dominated by viscous EHRDE. Using (29), (30), and (33) in (46), we get the time evolution of the total entropy for the case $a(t)=\left(t-t_{0}\right)^{\beta /(1-\alpha)}$ as

$$
\begin{aligned}
\dot{S}_{A} & +\dot{S}_{\mathrm{DE}}=\frac{1}{\beta^{2}(-1+\alpha+2 \beta)} 8 \pi^{2}\left(t-t_{0}\right)(-1+\alpha)^{2}(2 \\
& +\left(-t+t_{0}\right)(-1+\alpha)-2 \alpha \\
& -\frac{C_{2} e^{-\left(t-t_{0}\right)^{3}(-1+\alpha)^{2} / 9(-1+2 \alpha) \beta^{2} \eta}\left(t-t_{0}\right)^{2}(-1+\alpha)^{2}}{\beta}-3 \beta \\
& +3(-1+2 \alpha) \beta+\frac{9(-1+2 \alpha) \beta^{2}}{2(-1+\alpha)} \\
& +(-1+\alpha)\left(\frac{3(-1+2 \alpha) \beta^{2} \eta}{\left(t-t_{0}\right)^{2}(-1+\alpha)^{2}}\right) \\
& \left.+\frac{e^{-\left(t-t_{0}\right)^{3}(-1+\alpha)^{2} / 9(-1+2 \alpha) \beta^{2} \eta}\left(t-t_{0}\right)^{3}(-1+\alpha) E_{2 / 3}[T]}{2 \eta}\right) .
\end{aligned}
$$

Figure 11 shows that like the previous case $\dot{S}_{\text {total }}=\dot{S}_{A}+$ $\dot{S}_{\mathrm{DE}}>0$ and hence the GSL of thermodynamics is validated in a universe dominated by viscous EHRDE and expanding according to $a(t)=\left(t-t_{0}\right)^{\beta /(1-\alpha)}$. However, contrary to Figure 10, the time derivative of total entropy is increasing with evolution of the universe.

\section{Conclusions}

Motivated by $[30,43]$, we have presented a study on viscous extended holographic Ricci dark energy (EHRDE) in flat FRW universe based on Israel-Stewart approach. The work has been carried out in two phases. In one phase instead of choosing any specific form of scale factor we have reconstructed Hubble parameter $H$ based on the field equation (4) with $\rho=\rho_{\mathrm{DE}}=3 M_{p}^{2}\left(\alpha H^{2}+\beta \dot{H}\right)$ and subsequently solving the truncated Israel-Stewart theory given by $\tau \dot{\Pi}+\Pi=-3 H \xi$ for $\Pi$ we studied the behavior of EoS parameter $w_{\mathrm{DE}}$, bulk viscous pressure $\Pi$, state-finder parameters, and the thermodynamic consequences in terms of generalized second law (GSL) of thermodynamics. In this phase of study, we have observed the following.

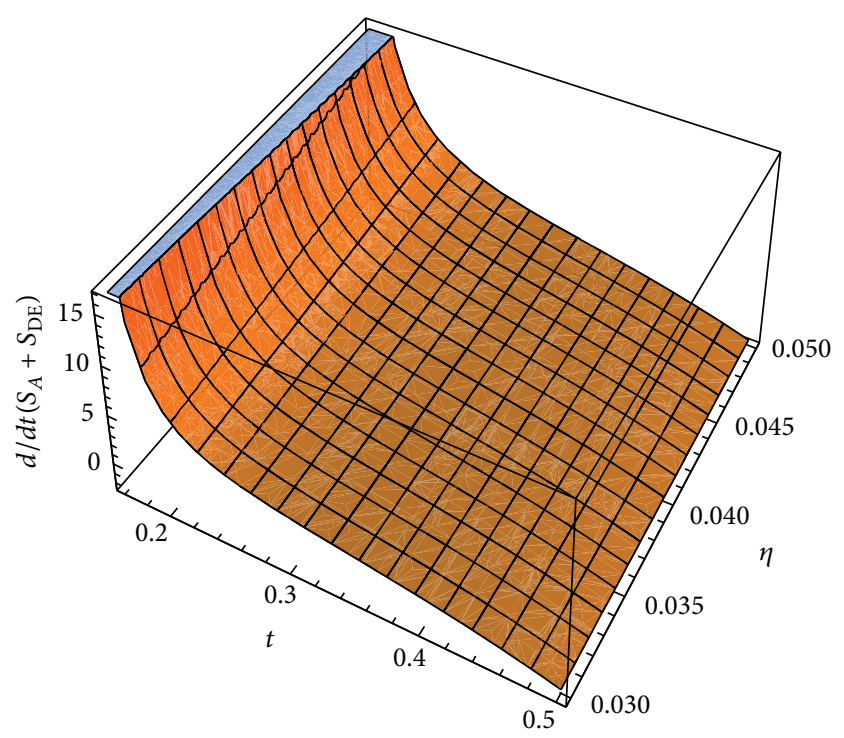

FIgURE 10: Plot of time evolution of total entropy $\left(\dot{S}_{A}+\dot{S}_{\mathrm{DE}}\right)$ based on (47). The $\{\alpha, \beta\}$ combination is taken as $\{0.9735,0.3701\}$ and $\eta$ ranges from 0.0002 to 0.0005 .

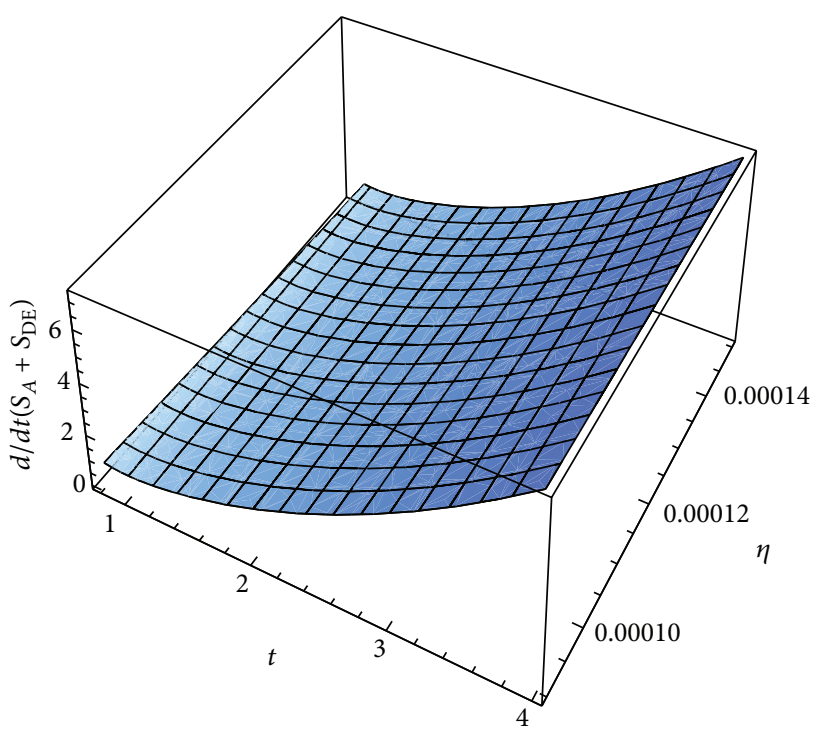

FIGURE 11: Plot of time evolution of total entropy $\left(\dot{S}_{A}+\dot{S}_{\mathrm{DE}}\right)$ based on (47) for $a(t)=\left(t-t_{0}\right)^{\beta /(1-\alpha)}$. The $\{\alpha, \beta\}$ combination is taken as $\{0.9180,0.3701\}$ and $\eta$ ranges from 0.00009 to 0.00015 .

Under the consideration that the universe is dominated by EHRDE, we have taken evolution equation for the bulk viscous pressure $\Pi$ in the framework of the truncated IsraelStewart theory as $\tau \dot{\Pi}+\Pi=-3 \xi H$, where $\tau$ is the relaxation time and $\xi$ is the bulk viscosity coefficient. Considering effective pressure as a sum of thermodynamic pressure of EHRDE and bulk viscous pressure, we have observed that under the influence of bulk viscosity the EoS parameter $w_{\mathrm{DE}}$ is behaving like phantom; that is, $w_{\mathrm{DE}} \leq-1$ (see Figure 1). Furthermore, it has been observed that the effect of bulk viscosity is not blowing up in the very late stage or very early 
stage of the universe. This observation is consistent with [43]. Some constraints have been derived for the model parameters $\alpha$ and $\beta$ and other constants. Obeying the observational studies that show that for EHRDE $\alpha=0.8502_{-0.0875-0.1064}^{+0.0984+0.1299}$ and $\beta=0.4817_{-0.0773-0.0955}^{+0.0842+1176}$ we have taken $\alpha=0.9735$, $\beta=0.3701$. It has been already mentioned that like [39] to obtain solution with Big Rip no restriction is imposed on $v$. Figure 1 shows that $w_{\mathrm{DE}}$ is decaying with evolution of the universe. It is observable that along with $\rho+p<0$ we also have $\rho+3 p<0$ with passage of cosmic time. Also, with passage of cosmic time $-(\rho+3 p)$ increases, and so all gravitationally bound systems will be dissociated [71] and the universe will end up in Big Rip. We have observed that the magnitude of the effective pressure $p_{\text {eff }}=p+\Pi$ is decaying with time (see Figure 2). Moreover, the nonnegative derivative of $\Pi$ (see Figure 3) has indicated that the effect of bulk viscosity is increasing with time. The bulk viscosity coefficient $\xi$ has been found to be an increasing function of time (see Figure 4). The state-finder parameters $\{r, s\}$ have also been studied. It has been observed that under the effect of bulk viscosity the state-finder trajectory $\{r-s\}$ for EHRDE is capable of reaching the $\Lambda$ CDM point, that is, $\{r=1, s=0\}$. We have further observed that, for finite $r, s \rightarrow-\infty$, which corresponds to dust phase. This shows that the viscous EHRDE interpolates between dust and $\Lambda$ CDM phases of the universe (see Figure 8 ). Finally we have studied the thermodynamics of the viscous EHRDE under the assumption that the universe is enveloped by apparent horizon. We have derived the expression of the time derivative of the total entropy and we have observed that (see Figure 10) the time derivative is positive throughout the evolution of the universe. This shows that the generalized second law of thermodynamics is valid for the viscous EHRDE.

In the next phase of the study, we have chosen the scale factor as $a(t)=\left(t-t_{0}\right)^{\beta /(1-\alpha)}$ and subsequently studied the cosmological parameters similar to that in the previous phase. The effective pressure and time derivative of bulk viscous pressure $\Pi$ appear to be of similar pattern to those in the earlier phase. However, the significant difference is observed in the case of EoS parameter $w_{\mathrm{DE}}$ (see Figure 5) and the statefinder trajectories $\{r-s\}$ (see Figure 9). The EoS parameter $w_{\mathrm{DE}}$ is found to cross the phantom barrier, that is, transiting from quintessence $\left(w_{\mathrm{DE}}>-1\right)$ to phantom $\left(w_{\mathrm{DE}}<-1\right)$. Thus, the model is found to behave like "Quintom" for the said form of scale factor. In the state-finder trajectory, unlike the previous case, the $\Lambda \mathrm{CDM}$ fixed point is not attainable and the model is deviated significantly from $\Lambda$ CDM. However, the dust phase is attainable like the previous case. Like the previous case, the GSL is found to be valid; that is, time derivative of the total entropy stays in the nonnegative level. However, contrary to what happened in the previous case, the time derivative of the total entropy is increasing with evolution of the universe.

\section{Competing Interests}

The author declares that there are no competing interests regarding the publication of this paper.

\section{Acknowledgments}

Financial support from DST, Government of India under Project Grant no. SR/FTP/PS-167/2011 is thankfully acknowledged. Warm hospitality provided by the Inter-University Centre for Astronomy and Astrophysics (IUCCA), Pune, India, during a scientific visit in May 2016 is duly acknowledged by the author.

\section{References}

[1] A. G. Riess, A. V. Filippenko, P. Challis et al., "Observational evidence from supernovae for an accelerating universe and a cosmological constant," The Astronomical Journal, vol. 116, no. 3, pp. 1009-1038, 1998.

[2] S. Perlmutter, G. Aldering, G. Goldhaber et al., "Measurements of $\Omega$ and $\Lambda$ from 42 high-redshift supernovae," The Astrophysical Journal, vol. 517, no. 2, article 565, 1999.

[3] J. A. Frieman, M. S. Turner, and D. Huterer, "Dark energy and the accelerating universe," Annual Review of Astronomy and Astrophysics, vol. 46, no. 1, pp. 385-432, 2008.

[4] E. J. Copeland, M. Sami, and S. Tsujikawa, "Dynamics of dark energy," International Journal of Modern Physics D, vol. 15, no. 11, pp. 1753-1935, 2006.

[5] K. Bamba, S. Capozziello, S. Nojiri, and S. D. Odintsov, "Dark energy cosmology: the equivalent description via different theoretical models and cosmography tests," Astrophysics and Space Science, vol. 342, no. 1, pp. 155-228, 2012.

[6] R. R. Caldwell and M. Kamionkowski, "The physics of cosmic acceleration," Annual Review of Nuclear and Particle Science, vol. 59, no. 1, pp. 397-429, 2009.

[7] S. Nojiri and S. D. Odintsov, "Unified cosmic history in modified gravity: from $F(R)$ theory to Lorentz non-invariant models," Physics Reports, vol. 505, no. 2-4, pp. 59-144, 2011.

[8] S. Nojiri and S. D. Odintsov, "Unifying phantom inflation with late-time acceleration: scalar phantom-non-phantom transition model and generalized holographic dark energy," General Relativity and Gravitation, vol. 38, no. 8, pp. 1285-1304, 2006.

[9] M. Li, "A model of holographic dark energy," Physics Letters B, vol. 603, no. 1-2, pp. 1-5, 2004.

[10] E. Elizalde, S. Nojiri, S. D. Odintsov, and P. Wang, "Dark energy: vacuum fluctuations, the effective phantom phase, and holography," Physical Review D, vol. 71, Article ID 103504, 2005.

[11] S. Nojiri and S. D. Odintsov, "Unifying phantom inflation with late-time acceleration: scalar phantom-non-phantom transition model and generalized holographic dark energy," General Relativity and Gravitation, vol. 38, no. 8, pp. 1285-1304, 2006.

[12] S. del Campo, J. C. Fabris, R. Herrera, and W. Zimdahl, "Holographic dark-energy models," Physical Review D, vol. 83, Article ID 123006, 2011.

[13] J.-L. Cui and J.-F. Zhang, "Comparing holographic dark energy models with statefinder," The European Physical Journal C, vol. 74, article 2849, 2014.

[14] S. Chattopadhyay, A. Jawad, and S. Rani, "Holographic polytropic $f(T)$ gravity models," Advances in High Energy Physics, vol. 2015, Article ID 798902, 15 pages, 2015.

[15] S. Chattopadhyay and A. Pasqua, "Consequences of holographic scalar field dark energy models in chameleon Brans-Dicke cosmology," in XXI DAE-BRNS High Energy Physics Symposium, B. Bhuyan, Ed., vol. 174 of Springer Proceedings in Physics, pp. 487-492, 2016. 
[16] Q.-G. Huang and Y. Gong, "Supernova constraints on a holographic dark energy model," Journal of Cosmology and Astroparticle Physics, vol. 2004, no. 8, p. 6, 2004.

[17] Q.-G. Huang and M. Li, "Anthropic principle favours the holographic dark energy," Journal of Cosmology and Astroparticle Physics, vol. 2005, no. 3, article 001, 2005.

[18] X. Zhang and F.-Q. Wu, "Constraints on holographic dark energy from the latest supernovae, galaxy clustering, and cosmic microwave background anisotropy observations," Physical Review D, vol. 76, Article ID 023502, 2007.

[19] L. N. Granda and A. Oliveros, "Infrared cut-off proposal for the holographic density," Physics Letters B, vol. 669, no. 5, pp. 275277, 2008.

[20] Y. U. Fei and Z. Jing-Fei, "Statefinder diagnosis for the extended holographic Ricci dark energy model without and with interaction," Communications in Theoretical Physics, vol. 59, no. 2, pp. 243-248, 2013.

[21] S. Chattopadhyay, "Extended holographic Ricci dark energy in chameleon Brans-Dicke cosmology," ISRN High Energy Physics, vol. 2013, Article ID 414615, 7 pages, 2013.

[22] R. Brustein and G. Veneziano, "Causal entropy bound for a spacelike region," Physical Review Letters, vol. 84, no. 25, pp. 5695-5698, 2000.

[23] C. Gao, F. Wu, X. Chen, and Y.-G. Shen, "Holographic dark energy model from Ricci scalar curvature," Physical Review D, vol. 79, no. 4, Article ID 043511, 7 pages, 2009.

[24] S. del Campo, J. C. Fabris, R. Herrera, and W. Zimdahl, "Cosmology with Ricci dark energy," Physical Review D, vol. 87, no. 12, Article ID 123002, 2013.

[25] S. Chattopadhyay, "On the various aspects of interacting Ricci dark energy and tachyonic field," Astrophysics and Space Science, vol. 331, no. 2, pp. 651-655, 2011.

[26] P. George and T. K. Mathew, "Holographic Ricci dark energy as running vacuum," Modern Physics Letters A: Particles and Fields, Gravitation, Cosmology, Nuclear Physics, vol. 31, no. 13, Article ID 1650075, 12 pages, 2016.

[27] C.-J. Feng, "Statefinder diagnosis for Ricci dark energy," Physics Letters B, vol. 670, no. 3, pp. 231-234, 2008.

[28] T. K. Mathew, J. Suresh, and D. Divakaran, "Modified holographic Ricci dark energy model and statefinder diagnosis in flat universe," International Journal of Modern Physics D, vol. 22, no. 9, Article ID 1350056, 17 pages, 2013.

[29] Y. Wang and L. Xu, "Current observational constraints to the holographic dark energy model with a new infrared cutoff via the Markov chain Monte Carlo method," Physical Review D, vol. 81, no. 8, 11 pages, 2010.

[30] C. S. J. Pun, L. Á. Gergely, M. K. Mak, Z. Kovács, G. M. Szabó, and T. Harko, "Viscous dissipative Chaplygin gas dominated homogenous and isotropic cosmological models," Physical Review D, vol. 77, no. 6, Article ID 063528, 2008.

[31] L. P. Chimento, A. S. Jakubi, and D. Pavón, "Enlarged quintessence cosmology," Physical Review D, vol. 62, no. 6, Article ID 063508, 9 pages, 2000.

[32] C. Eckart, "The thermodynamics of irreversible processes. III. Relativistic theory of the simple fluid," Physical Review, vol. 58, no. 10, pp. 919-924, 1940.

[33] L. D. Landau and E. M. Lifshitz, Fluid Mechanics, Butterworth Heinemann, Oxford, UK, 1987.

[34] W. Israel and J. M. Stewart, "Thermodynamics of nonstationary and transient effects in a relativistic gas," Physics Letters A, vol. 58, no. 4, pp. 213-215, 1976.
[35] S. Nojiri and S. D. Odintsov, "Inhomogeneous equation of state of the universe: phantom era, future singularity, and crossing the phantom barrier," Physical Review D, vol. 72, no. 2, Article ID 023003, 12 pages, 2005.

[36] H. B. Benaoum, "Modified Chaplygin gas cosmology with bulk viscosity," International Journal of Modern Physics D, vol. 23, no. 10, Article ID 1450082, 2014.

[37] J. Naji, S. Heydari, and R. Darabi, "New version of viscous Chaplygin gas cosmology with varying gravitational constant," Canadian Journal of Physics, vol. 92, no. 12, pp. 1556-1561, 2014.

[38] K. Karimiyan and J. Naji, "Interacting viscous modified Chaplygin gas cosmology in presence of cosmological constant," International Journal of Theoretical Physics, vol. 53, no. 7, pp. 2396-2403, 2014.

[39] M. Cataldo, N. Cruz, and S. Lepe, "Viscous dark energy and phantom evolution," Physics Letters B, vol. 619, no. 1-2, pp. 5-10, 2005.

[40] I. Brevik and O. Gorbunova, "Dark energy and viscous cosmology," General Relativity and Gravitation, vol. 37, no. 12, pp. 20392045, 2005.

[41] J. Ren and X.-H. Meng, "Cosmological model with viscosity media (dark fluid) described by an effective equation of state," Physics Letters B, vol. 633, no. 1, pp. 1-8, 2006.

[42] M. R. Setare and A. Sheykhi, "Viscous dark energy and generalized second law of thermodynamics," International Journal of Modern Physics D, vol. 19, no. 7, pp. 1205-1215, 2010.

[43] C.-J. Feng and X.-Z. Li, "Viscous Ricci dark energy," Physics Letters B, vol. 680, no. 4, pp. 355-358, 2009.

[44] H. Amirhashchi and A. Pradhan, "Viscous dark energy and phantom field in an anisotropic universe," Astrophysics and Space Science, vol. 351, no. 1, pp. 59-65, 2014.

[45] I. Brevik, V. V. Obukhov, and A. V. Timoshkin, "Dark energy coupled with dark matter in viscous fluid cosmology," Astrophysics and Space Science, vol. 355, no. 2, pp. 399-403, 2015.

[46] H. Velten, J. Wang, and X. Meng, "Phantom dark energy as an effect of bulk viscosity," Physical Review D, vol. 88, Article ID 123504, 2013.

[47] M. Jamil and M. U. Farooq, "Interacting holographic viscous dark energy model," International Journal of Theoretical Physics, vol. 49, article 42, 2010.

[48] M. R. Setare and V. Kamali, "Cosmological perturbations in warm-tachyon inflationary universe model with viscous pressure on the brane," Journal of High Energy Physics, vol. 2013, article 66, 2013.

[49] K. Bamba and S. D. Odintsov, "Inflation in a viscous fluid model," The European Physical Journal C, vol. 76, p. 18, 2016.

[50] I. Brevik, S. Nojiri, S. D. Odintsov, and L. Vanzo, "Entropy and universality of the Cardy-Verlinde formula in a dark energy universe," Physical Review D, vol. 70, no. 4, Article ID 043520, 2004.

[51] I. Brevik, S. Nojiri, S. D. Odintsov, and D. Sáez-Gómez, “CardyVerlinde formula in FRW Universe with inhomogeneous generalized fluid and dynamical entropy bounds near the future singularity," The European Physical Journal C, vol. 69, no. 3-4, pp. 563-574, 2010.

[52] M. Sharif and S. Rani, "Viscous dark energy in $f(T)$ gravity," Modern Physics Letters A, vol. 28, no. 27, Article ID 1350118, 13 pages, 2013.

[53] P. Kumar and C. P. Singh, "Viscous cosmology with matter creation in modified $f(R, T)$ gravity," Astrophysics and Space Science, vol. 357, no. 2, article 120, 2015. 
[54] V. R. Chirde and S. H. Shekh, "Barotropic bulk viscous FRW cosmological model in teleparallel gravity," Bulgarian Journal of Physics, vol. 41, no. 4, pp. 258-273, 2014.

[55] R. Colistete Jr., J. C. Fabris, J. Tossa, and W. Zimdahl, "Bulk viscous cosmology," Physical Review D, vol. 76, no. 10, Article ID 103516, 2007.

[56] V. Sahni, T. D. Saini, A. A. Starobinsky, and U. Alam, "Statefinder-a new geometrical diagnostic of dark energy," JETP Letters, vol. 77, no. 5, pp. 201-206, 2003.

[57] U. Alam, V. Sahni, T. D. Saini, and A. A. Starobinsky, "Exploring the expanding Universe and dark energy using the statefinder diagnostic," Monthly Notices of the Royal Astronomical Society, vol. 344, no. 4, pp. 1057-1074, 2003.

[58] M. Jamil, D. Momeni, R. Myrzakulov, and P. Rudra, "Statefinder analysis of $f(T)$ cosmology," Journal of the Physical Society of Japan, vol. 81, Article ID 114004, 2012.

[59] W. Zimdahl and D. Pavón, "Statefinder parameters for interacting dark energy," General Relativity and Gravitation, vol. 36, no. 6, pp. 1483-1491, 2004.

[60] C.-J. Feng, "Statefinder diagnosis for Ricci dark energy," Physics Letters B, vol. 670, pp. 231-234, 2008.

[61] J. D. Bekenstein, "Black holes and the second law," Lettere Al Nuovo Cimento, vol. 4, no. 15, pp. 737-740, 1972.

[62] J. D. Bekenstein, "Black holes and entropy", Physical Review D, vol. 7, pp. 2333-2346, 1973.

[63] J. D. Bekenstein, "Generalized second law of thermodynamics in black-hole physics," Physical Review D, vol. 9, no. 12, pp. 32923300, 1974.

[64] S. Chakraborty, "Generalized Bekenstein-Hawking system: logarithmic correction," The European Physical Journal C, vol. 74, article 2876, 2014.

[65] S. Hod, "Bekenstein's generalized second law of thermodynamics: the role of the hoop conjecture," Physics Letters B, vol. 751, pp. 241-245, 2015.

[66] H. Moradpour and R. Dehghani, "Thermodynamical study of FRW universe in quasi-topological theory," Advances in High Energy Physics, vol. 2016, Article ID 7248520, 10 pages, 2016.

[67] R. Bousso and N. Engelhardt, "Generalized second law for cosmology," Physical Review D, vol. 93, no. 2, Article ID 024025, 2016.

[68] M. R. Setare, "Generalized second law of thermodynamics in quintom dominated universe," Physics Letters B, vol. 641, no. 2, pp. 130-133, 2006.

[69] K. Bamba, M. Jamil, D. Momeni, and R. Myrzakulov, "Generalized second law of thermodynamics in $f(T)$ gravity with entropy corrections," Astrophysics and Space Science, vol. 344, no. 1, pp. 259-267, 2013.

[70] R. Herrera and N. Videla, "The generalized second law of thermodynamics for interacting $\mathrm{f}(\mathrm{R})$ gravity," International Journal of Modern Physics D, vol. 23, no. 8, Article ID 1450071, 16 pages, 2014.

[71] R. R. Caldwell, M. Kamionkowski, and N. N. Weinberg, "Phantom energy: dark energy with $w<-1$ causes a cosmic doomsday," Physical Review Letters, vol. 91, no. 7, Article ID 071301, 4 pages, 2003. 

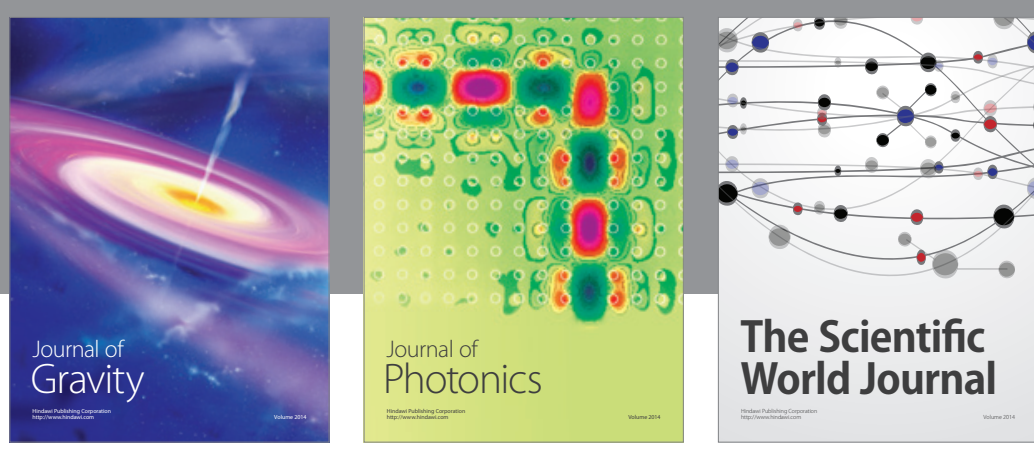

The Scientific World Journal
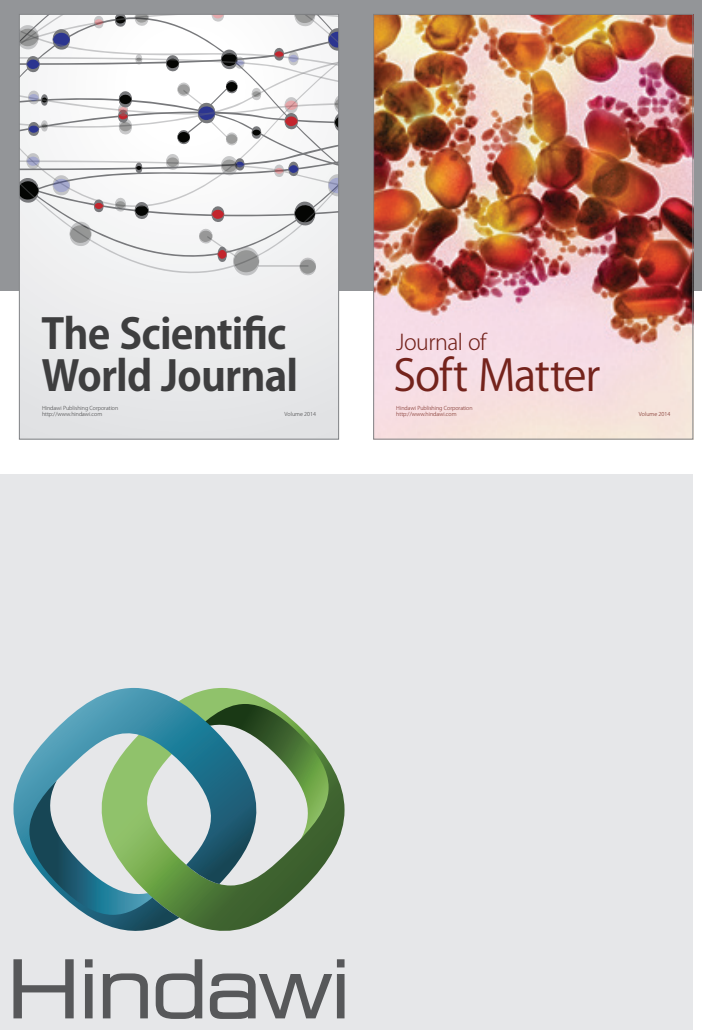

Submit your manuscripts at

http://www.hindawi.com

nternational Journal of

Statistical Mechanics
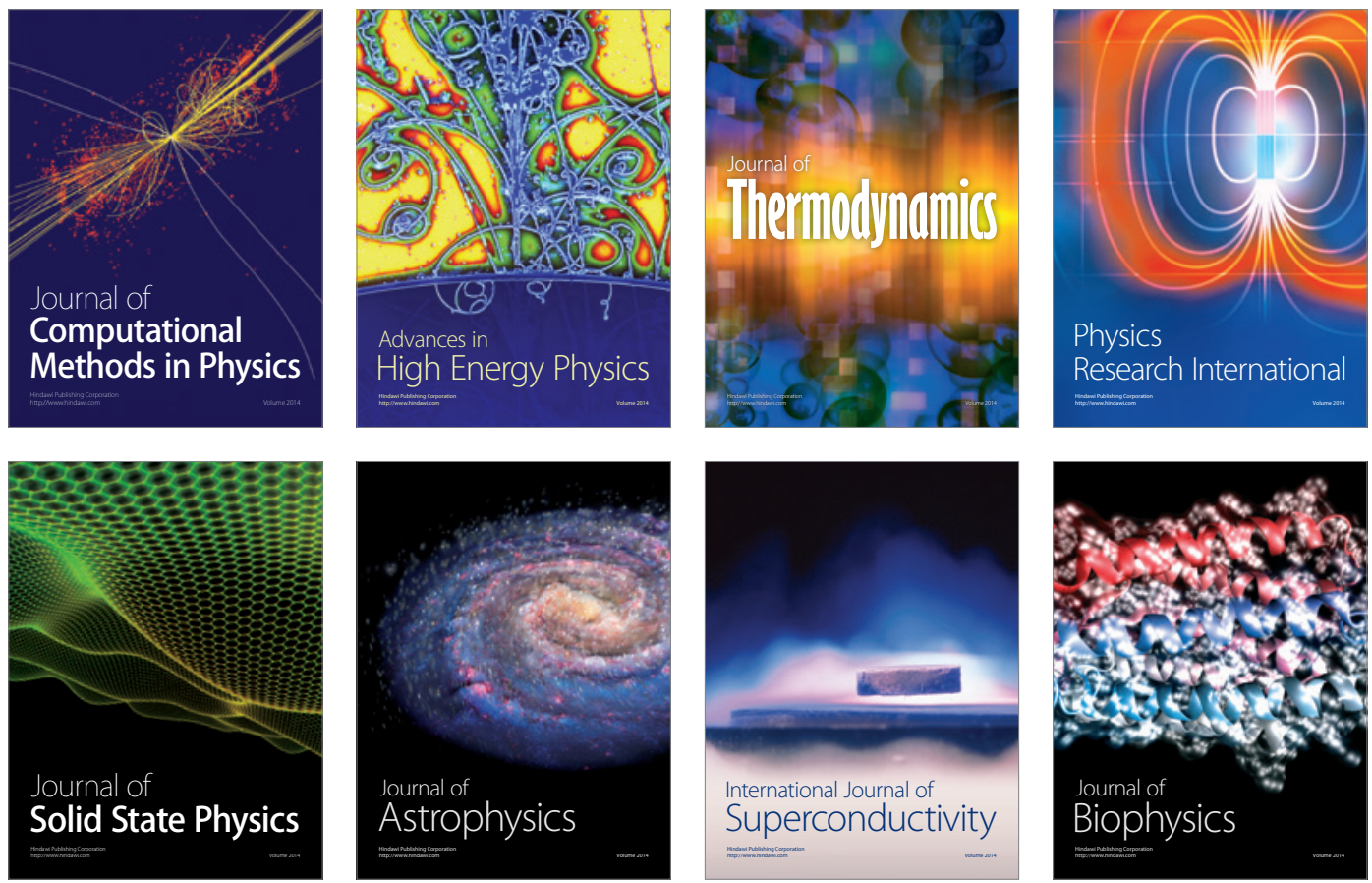
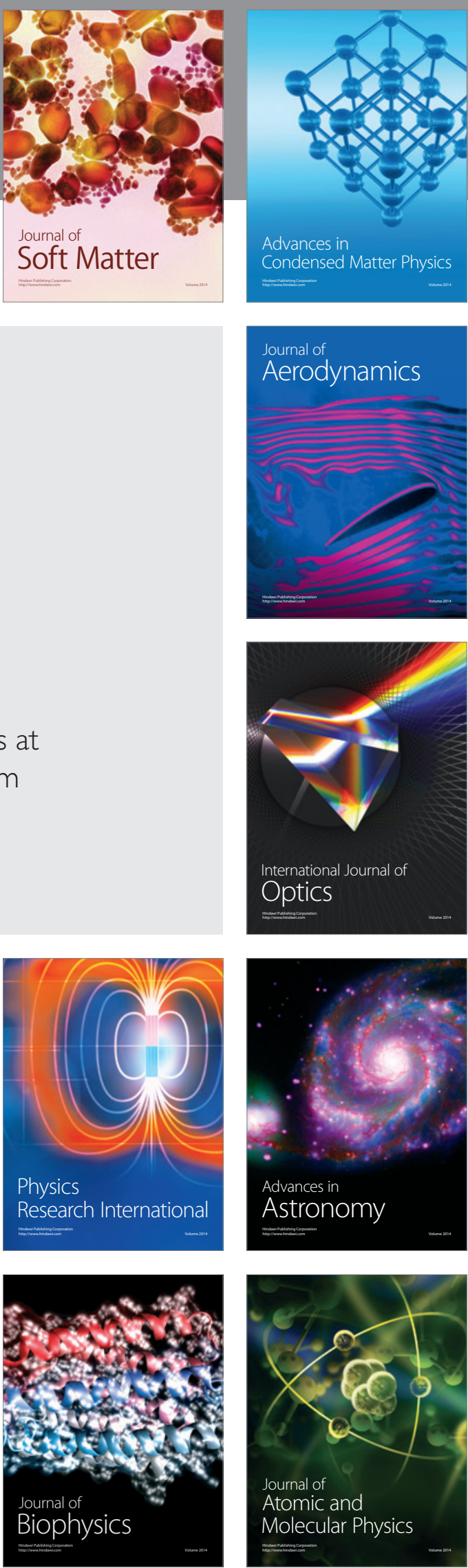